\title{
An enhanced pseudo-3D model for hydraulic fracturing accounting for viscous height growth, non-local elasticity, and lateral toughness
}

\author{
E.V. Dontsov and A.P. Peirce \\ Department of Mathematics, University of British Columbia, Vancouver, BC, V6T 1Z2, Canada \\ Email address for correspondence: peirce@math.ubc.ca, phone: +1-604-822-2104
}

\begin{abstract}
The goal of this paper is to develop an enhanced pseudo-3D (P3D) model for hydraulic fracturing (HF), whose predictions are more accurate compared to that of the original P3D model, but which requires significantly less computational resources than a fully planar HF simulator. We show that the lack of viscous resistance in the height growth and the local approximation in the computation of elastic interactions, which precludes the incorporation of lateral toughness, are the primary weaknesses of the original P3D model that considers symmetric stress barriers and no leak-off. To account for the viscous resistance, an apparent fracture toughness is introduced. The apparent toughness is calibrated using a one-dimensional HF model resulting in an approximate expression that captures all regimes of propagation. To incorporate non-local elastic interactions, the fracture opening in every vertical cross-section is approximated by a plane-strain solution, and then the 2D elasticity interaction integral is evaluated. To increase the computational efficiency, this 2D integral is further approximated by two one-dimensional integrals. The use of non-local elasticity allows us to include the asymptotic solution in the tip element, and, in particular, to include the effect of lateral fracture toughness. To further increase the accuracy of the P3D model, the flat fracture tip is replaced by its curved counterpart. This also permits us to capture radial behaviour at early times before the fracture has reached the stress barriers. To evaluate the accuracy of the model we have developed, the results are compared to the predictions calculated using a recently developed fully planar HF simulator, which is able to capture viscous, toughness, and intermediate propagation regimes. It is shown that the enhanced P3D model is able to approximate the propagation of hydraulic fractures accurately for various regimes of propagation, as well as for different fracture aspect ratios.
\end{abstract}

Keywords: Hydraulic fracturing, pseudo-3D model.

\section{Introduction}

Hydraulic fracturing (HF) is a process in which a pressurized fluid is injected into rock to cause fracture initiation and propagation. Industrial applications of HF include accelerating the waste remediation process [1], waste disposal [2], preconditioning in rock mining [3], and oil and gas reservoir stimulation [4], where the latter stands out as one of the most common applications.

To successfully apply HF, the treatment should be designed using an appropriate HF model or numerical simulator. The earliest models started with the simplest one-dimensional Khristianovich-Zheltov-GeertsmaDe Klerk (KGD) model [5], in which the fracture propagates under plane strain conditions and the coupling between viscous fluid flow and elasticity is included. Later, the Perkins-Kern-Nordgren (PKN) model [6, 7] was developed to simulate fracture propagation in a horizontally layered medium. The PKN model assumes that the fracture propagates in the horizontal direction (i.e. along the reservoir layer) and has a constant height that is equal to the thickness of the reservoir layer. Each vertical cross-section is assumed to be elliptic with a uniform fluid pressure, where the latter is calculated assuming that a state of plane strain prevails in each vertical plane. The primary advantage of the PKN model lies in its computational efficiency, since averaging over the vertical direction reduces the calculations to solving a one-dimensional problem. 
To allow for height growth, the pseudo-3D (P3D) model has been developed $[8,9,10]$ as an extension of the PKN model. There have been multiple variations of the P3D model. In particular, the geometry of the fracture was either approximated by ellipses or the fracture's lateral dimension was divided into elements, where each element had a specific height. The first case corresponds to the so-called lumped P3D model $[11,12]$, while the second variation is called a cell-based P3D model [9, 10]. In addition, two types of height growth mechanisms were used: i) equilibrium height growth [9], where it is assumed that the pressure is uniform and a toughness propagation criterion is used to calculate the fracture height; ii) dynamic height growth [10], where the height is calculated from the solution of a KGD problem (or its approximation) for each vertical cross-section. With the advent of more computational power, scientific effort has shifted towards developing more accurate planar 3D models (PL3D) $[13,14,15]$. In such models, the fracture footprint is assumed to be planar and is discretized using a two-dimensional mesh. Two-dimensional fluid flow and elastic interactions between all elements are then considered. These modifications significantly increase the accuracy of HF simulators, but, at the same time, the CPU time increases dramatically. The reason for this lies in the fact that both the PKN and P3D models require the solution of essentially a one-dimensional lubrication problem with a local relation describing elastic interactions, while the PL3D models involve coupling between the two-dimensional lubrication operator and the two-dimensional elasticity operator representing full non-local interactions. A more detailed review of various HF simulators can be found in [16]. Motivated by recent developments in the petroleum industry and the further development of computational power, researchers have started to investigate the interaction between multiple hydraulic fractures. As an example, the so-called phenomenon of stress shadowing [17] is investigated in [18]. Stress shadowing occurs when one attempts to grow multiple hydraulic fractures simultaneously. The mutual elastic interactions produce compressive stresses that affect the evolution of neighbouring fractures and result in dissimilar growth of fractures initiated from the different perforations in a stage. Finally, the complexity of the hydraulic fracturing problem can be increased even further by considering non-planar fractures [19].

From the above discussion we observe that the complexity of hydraulic fracture modelling has increased with the advent of more computational power and the desire for models that represent real HF more accurately. Although more complicated models allow us to obtain accurate results, there are situations in which the required computational time imposes severe limitations on the numerical experiments that can feasibly be performed. In this situation, a computationally efficient and accurate P3D model, which allows us to obtain results rapidly, becomes an appropriate choice. As an example, the P3D model is used in $[20,21]$ for production optimization purposes. Also, hydraulic fracture interaction is investigated using a P3D modelling approach in [22]. In the latter case, the use of a P3D model permits the analysis of complex phenomena without using excessive computational resources. Unfortunately, despite having the advantage in computational time, P3D models are not accurate when the assumptions of the model are not met. In particular, since viscous losses in the vertical direction are disregarded in a P3D model with equilibrium height growth, fracture height estimates are inaccurate when the value of fracture toughness is small. Likewise, owing to the local elasticity assumption, the P3D model can be inaccurate for large values of fracture toughness, for which lateral fracture propagation occurs in the toughness regime. Recognizing the problem, corrections to the P3D model, intended to increase the accuracy, have been made. Two-dimensional fluid flow has been incorporated into a P3D model [23] making it possible to capture fracture height growth more accurately. The work by [12] also considers two-dimensional fluid flow, but approximates the fracture geometry by ellipses, which makes it possible to capture the effect of fracture toughness, but noticeably restricts the geometry of the hydraulic fracture. With regard to the PKN model, [24] mentions unpublished work, in which a toughness propagation criterion for PKN fractures is introduced. Also, a one-dimensional non-local elasticity equation for PKN fractures is derived and analyzed in [25]. The primary purpose of that work was to introduce a non-local relation between the fluid pressure and fracture opening, which can be further used to develop PKN-based HF models that can capture fracture toughness in the lateral direction.

Recognizing the necessity to develop a more accurate P3D model, this paper aims to introduce an enhanced P3D (EP3D) model, which accounts for viscous height growth, non-local elasticity, and fracture toughness in the lateral direction. The developments are based on the cell-based P3D model with equilibrium height growth, analyzed in [26], and assume no leak-off (i.e. that the rock is impermeable). Correction for viscous height growth is implemented through the introduction of an apparent toughness, which ap- 
proximately captures the viscous dissipation in the vertical direction. This provides a way of correcting the vertical growth (an alternative to [23]), which is applicable to a P3D model with equilibrium height growth. Following the developments in [25], non-local elasticity is incorporated in a P3D model and is then implemented into a HF simulator. This allows us to capture the effect of lateral fracture toughness with a P3D model. Moreover, since the EP3D model still relies on the solution of a one-dimensional problem, it permits us to obtain results extremely rapidly.

The paper is organized as follows. Section 2 briefly describes the classic P3D model with symmetric stress barriers and equilibrium height growth, as well as its limitations. Section 3 incorporates the effect of viscous resistance in the vertical direction by calibrating the model using a one-dimensional KGD model. Then, Section 4 describes a procedure to implement non-local elasticity into a P3D model. Section 5 introduces a curved fracture tip, which also enables us to accurately capture radially symmetric behaviour at early stages in the HF evolution. The details of the numerical implementation are presented in an appendix.

\section{Limitations of classical pseudo-3D model with equilibrium height growth}

To motivate the necessity for improvements to the original pseudo-3D (P3D) model, this section aims first, to briefly describe the P3D model with its assumptions, and then to compare the model predictions to the output of a recently developed fully-planar hydraulic fracturing simulator that is able to capture the multiscale behaviour encountered when the processes of viscous dissipation and toughness energy release compete. This allows us to highlight the weak points of the P3D model that can lead to noticeable errors for some problem parameters.

\subsection{Pseudo-3D (P3D) model}

The P3D model under consideration assumes that the reservoir layer with height $H$ is surrounded by two symmetric stress barriers that impose an additional confining stress $\Delta \sigma$, as shown in Fig. 1 . For this problem geometry, a fracture grows predominantly in the horizontal direction (i.e. along the $x$ axis), but height growth in the vertical direction is also allowed. The original P3D model formulated in [26] is based on the following assumptions: i) the lateral fracture tip is vertical and its height is equal to $H$, ii) the vertical component of the flux is negligible compared to the horizontal omcponent, which implies that the pressure is constant along the $z$ axis, iii) plane strain elasticity conditions apply for any vertical $(y, z)$ plane, iv) leak-off is limited only to the reservoir layer (i.e. no leak-off in the stress barriers) and behaves according to the Carter model [27]. The assumptions of plane-strain elasticity and uniform pressure in each $(y, z)$ plane
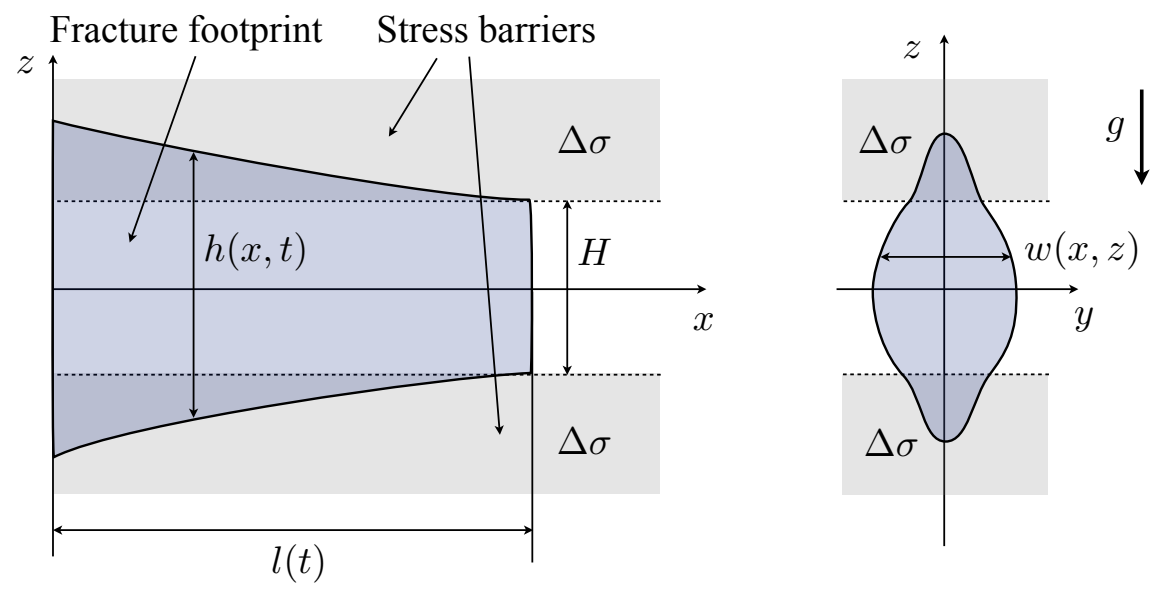

Figure 1: Schematics of the P3D fracture. 
allow us to find a solution for the fracture width in each cross-section as

$$
w(x, z)=\frac{2}{E^{\prime}}(p(x)-\Delta \sigma) \chi+\frac{4 \Delta \sigma}{\pi E^{\prime}}\left\{\chi \arcsin \left(\frac{H}{h}\right)-z \ln \left|\frac{H \chi+2 z \psi}{H \chi-2 z \psi}\right|+\frac{H}{2} \ln \left|\frac{\chi+\psi}{\chi-\psi}\right|\right\}
$$

where $w(x, z)$ is a fracture width, $\Delta \sigma$ is the amplitude of the stress barrier, $h$ denotes fracture height (see Fig. 1), $p(x)$ is the fluid pressure, $\chi=\sqrt{h^{2}-4 z^{2}}, \psi=\sqrt{h^{2}-H^{2}}$, while $E^{\prime}=E /\left(1-\nu^{2}\right)$ is the plane strain Young's modulus. The use of a constant pressure in the vertical direction implies that the fracture propagates in the toughness regime. In this case, the fracture propagation criterion $K_{I}=K_{I c}$ can be used to find

$$
p=\Delta \sigma\left[1+\sqrt{\frac{2}{\pi H}} \frac{K_{I c}}{\Delta \sigma} \sqrt{\frac{H}{h}}-\frac{2}{\pi} \arcsin \left(\frac{H}{h}\right)\right],
$$

where $K_{I}$ is the stress intensity factor and $K_{I c}$ is the fracture toughness. Equations (1) and (2) can be combined to yield

$$
w(x, z)=\frac{2}{E^{\prime}} \sqrt{\frac{2}{\pi h}} K_{I c} \chi+\frac{4 \Delta \sigma}{\pi E^{\prime}}\left\{-z \ln \left|\frac{H \chi+2 z \psi}{H \chi-2 z \psi}\right|+\frac{H}{2} \ln \left|\frac{\chi+\psi}{\chi-\psi}\right|\right\},
$$

where the first term represents the fracture opening due to the fracture toughness, and the second terms capture the effect of the stress barriers. The aforementioned formulas (3) and (2) apply in the regions where $h>H$. In situations when $h=H$, an elliptic fracture width profile is used instead of (3), i.e. $w=2\left(E^{\prime}\right)^{-1} \chi p(x)$. The primary computational advantage of the P3D model lies in the fact that it can be formulated in terms of an effective width, defined as

$$
\bar{w}=\frac{1}{H} \int_{-h / 2}^{h / 2} w d z .
$$

Relation (3) can be integrated to obtain

$$
\bar{w}=\frac{H}{E^{\prime}}\left(\sqrt{\frac{\pi}{2 H}} K_{I c}\left(\frac{h}{H}\right)^{3 / 2}+\Delta \sigma \sqrt{\frac{h^{2}}{H^{2}}-1}\right), \quad h>H,
$$

which can be inverted to yield the function $h(\bar{w})$. If one uses this result, then the fracture width becomes a function of the effective width, i.e. $w=w(\bar{w})$. In other words, the knowledge of $\bar{w}(x)$ is sufficient to obtain the fracture width $w(x, z)$. This makes the problem one-dimensional and computationally efficient. The governing equation, that represents the vertically-integrated lubrication equation, can be written as

$$
\frac{\partial \bar{w}}{\partial t}+\frac{\partial \bar{q}_{x}}{\partial x}+\frac{C^{\prime}}{\sqrt{t-t_{0}(x)}}=\frac{Q_{0}}{H} \delta(x),
$$

where the averaged flux is given by

$$
\bar{q}_{x}=-\frac{1}{H \mu^{\prime}} \frac{\partial p}{\partial x} \int_{-h / 2}^{h / 2} w^{3} d z
$$

Here $C^{\prime}=2 C_{L}$ is Carter's leak-off coefficient, $Q_{0}$ is the total fluid volume pumped into the fracture per unit time, $t_{0}(x)$ is the inverse of the fracture length function $x=l(t)$, and $\mu^{\prime}=12 \mu$, where $\mu$ is the fracturing fluid viscosity. Given (2)-(5) and (7), equation (6) can be solved numerically (see e.g. [26]) for $\bar{w}(x)$, which can then be used to find the fracture opening $w(x, z)$, the height growth $h(x)$, and the fluid pressure $p(x)$.

\subsection{Drawbacks of the P3D model}

While the computational time associated with the P3D model is not an issue, the accuracy and applicability range have limitations. As was mentioned in the introduction, this paper deals with fracture 
propagation in an impermeable medium (no leak-off), so, of the four P3D model assumptions, only the first three are relevant, namely: that the lateral fracture tip is vertical; that the pressure is constant in vertical cross sections; and that plane strain elastic conditions apply in any vertical plane $(y, z)$.

In the absence of leak-off, there are two dissipative mechanisms that control the fracture geometry: the viscous dissipation and the energy release due to formation of new fracture surface. If the viscous dissipation is dominant, then the fracture propagates in the viscous regime, otherwise it propagates in the toughness regime. The assumptions of the P3D model imply that the fracture propagates in the toughness regime in the vertical direction, while, since the fracture toughness in the lateral direction is not accounted for, the fracture is assumed to propagate in the viscous regime in the horizontal direction. This means that out of the two possible dissipative mechanisms, only one is considered in the vertical direction and, similarly, only one is considered in the horizontal direction. In situations when the fracture toughness is small, or even zero, vertical height growth will be overestimated by the classic P3D model, since viscous resistance in the vertical direction, which should limit the fracture height, is ignored by the model. At the same time, for pay zones with a large fracture toughness or at advanced times, the propagation in the horizontal direction may become toughness-dominated. In this case, the P3D model will overestimate the fracture length as the local elasticity assumption precludes accounting for the resistance due to fracture toughness when determining the progress of the fracture front in the horizontal direction. Determining the bounds on the fracture toughness, for which the P3D model is valid, is a challenge, since hydraulic fractures are known [28] to change their regime of propagation with time. Thus the bounds on the fracture toughness, within which the P3D model is valid, are time-dependent. As a result, in spite of using the same problem parameters, the P3D model may be reasonably accurate over some time intervals and completely wrong over others.

To illustrate the existence of a validity region for the P3D model and to highlight its limitations, Fig. 2 compares the results of the P3D model with those obtained using the fully-planar Implicit Level Set Algorithm (ILSA) scheme $[15,29]$. The parameters for the computation are chosen as $H=0.05 \mathrm{~m}, \mu=30.2 \mathrm{~Pa} \cdot \mathrm{s}$, $\nu=0.4, E=3.3 \mathrm{GPa}, Q_{0}=1.7 \mathrm{~mm}^{3} / \mathrm{s}, \Delta \sigma=4.3 \mathrm{MPa}$. The rock is assumed impermeable in both models (i.e. no leak-off). Three values of fracture toughness are considered, $K_{I c}=0, K_{I c}=0.16 \mathrm{MPa} \cdot \mathrm{m}^{1 / 2}$, and $K_{I c}=0.94 \mathrm{MPa} \cdot \mathrm{m}^{1 / 2}$. The results of the simulations are compared at time instant $t=604 \mathrm{~s}$. Note that these parameters (except for those with fracture toughness) correspond to the laboratory experiments in [30]. Fig. 2 compares the fracture footprints (top picture), the variation of the fracture width and pressure versus $x$ at $z=0$ (middle and bottom left pictures), and the variation of the fracture width and pressure versus $z$ at $x=0$ (middle and bottom right pictures). The stress barrier is indicated by a thick black line. The ILSA results are shown by solid lines, while the P3D data is shown by dashed lines. Blue lines correspond to the viscous regime, i.e. $K_{I c}=0$, red lines correspond to the toughness regime $K_{I c}=0.94 \mathrm{MPa}$, while magenta lines represent the intermediate case with $K_{I c}=0.16 \mathrm{MPam}^{1 / 2}$. As can be seen from the figure, the P3D model is the most accurate for the intermediate case with $K_{I c}=0.16 \mathrm{MPam}^{1 / 2}$. For this moderate fracture toughness, the fracture length is accurately captured by the viscous regime lateral growth mode that is built into the P3D model, but the height growth is overestimated. The P3D solution for $K_{I c}=0.94 \mathrm{MPam}^{1 / 2}$ provides very poor estimates of all characteristics of the fracture, namely, the fracture length, the height, the opening, and the pressure. With regard to the overestimated height growth, it is interesting to observe that the ILSA results are nearly identical for $K_{I c}=0$ and $K_{I c}=0.16 \mathrm{MPa} \cdot \mathrm{m}^{1 / 2}$. Since the P3D model is more accurate for $K_{I c}=0.16 \mathrm{MPa} \cdot \mathrm{m}^{1 / 2}$, it might be useful to approximate the viscous ILSA solution by a P3D solution with some apparent toughness. In other words, the dashed magenta lines (i.e. the P3D result for $K_{I c}=0.16 \mathrm{MPa} \cdot \mathrm{m}^{1 / 2}$ ) are close to both the viscous solution and the corresponding ILSA solution with $K_{I c}=0.16 \mathrm{MPa} \cdot \mathrm{m}^{1 / 2}$. For this reason, there is a possibility to introduce an effective or apparent toughness in the P3D model, which can capture viscous resistance in the vertical direction. The next section describes a procedure to calculate this apparent toughness. It is also possible to upgrade the P3D model to capture the solution for high values of toughness, e.g. $K_{I c}=0.94 \mathrm{MPa}$, for which the original model works poorly. One option to limit the lateral growth is to introduce a fracture toughness at the corresponding fracture tip in the P3D model. However, this is not sufficient. The tip element can possibly satisfy the fracture toughness growth criterion, but since local elasticity is used (i.e. plane strain conditions are assumed in each vertical cross-section), there will be no elastic interaction with the rest of the fracture. Thus, the effect of toughness will change the fracture shape only locally without affecting the global behaviour. To deal with 
this problem, it is necessary to introduce a non-local or full elastic interaction, as is done for planar 3D models. This inevitably leads to additional computational costs, but we demonstrate that this can be done efficiently. The problem of including full elastic interactions into the P3D model is addressed in Section 4.

\section{Correction for viscous resistance in the vertical direction}

\subsection{The concept}

As discussed in the previous section, one weakness of the P3D model is the lack of viscous resistance in the vertical direction, which causes the fracture height growth to be overestimated, as shown in Fig. 2 . At the same time, the comparison between the ILSA solutions for $K_{I c}=0$ and $K_{I c}=0.16 \mathrm{MPa} \cdot \mathrm{m}^{1 / 2}$ shows that a small value of fracture toughness does not significantly affect the fracture footprint, while the P3D solution changes significantly and is more accurate for $K_{I c}=0.16 \mathrm{MPa} \cdot \mathrm{m}^{1 / 2}$. This establishes that a purely viscous ILSA solution (i.e. with $K_{I c}=0$ ) is approximated better by a P3D with a small toughness. The remaining question is how to find the appropriate value for this apparent toughness to achieve the best approximation. One possibility is to equate the fracture width calculated according to the viscous and toughness asymptotic solutions at some distance $d$, in which case

$$
w_{K} \equiv \sqrt{\frac{32}{\pi}} \frac{\Delta K_{I c}}{E^{\prime}} d^{1 / 2}=\beta_{m}\left(\frac{\mu^{\prime} V}{E^{\prime}}\right)^{1 / 3} d^{2 / 3} \equiv w_{M},
$$

where $w_{K}$ and $w_{M}$ denote respectively the toughness and viscous asymptotic solutions, $\Delta K_{I c}$ is an apparent toughness, $\beta_{m}=2^{1 / 3} \cdot 3^{5 / 6}$, while

$$
V=\frac{1}{2} \frac{\partial h}{\partial t}
$$

is the speed of the horizontal fracture fronts at the top or bottom of the blade-like fracture. Equation (8) yields the following formula for the apparent toughness

$$
\Delta K_{I c}=\sqrt{\frac{\pi}{32}} \beta_{m} E^{\prime 2 / 3} \mu^{1 / 3} V^{1 / 3} d^{1 / 6} .
$$

One can also take an energetic approach and require a balance between the energy release rate for the toughness regime and the viscous dissipation for the viscous regime. If the fracture propagates with a velocity $V$, then the energy released per unit time for a mode $I$ crack is

$$
\frac{\partial U_{K}}{\partial t}=V \frac{\Delta K_{I c}^{2}}{E^{\prime}}
$$

At the same time, since the fluid velocity profile inside the fracture is $v(y)=\frac{3}{2} V\left(1-4 y^{2} / w^{2}\right)$, the total viscous losses are

$$
\frac{\partial U_{M}}{\partial t}=\int_{0}^{d} \int_{-w / 2}^{w / 2} \mu\left(\frac{\partial v}{\partial y}\right)^{2} d y d z=3 \beta_{m}^{-1} \mu^{\prime 2 / 3} E^{1 / 3} V^{5 / 3} d^{1 / 3},
$$

where the asymptotic solution for the fracture opening $w_{M}=\beta_{m}\left(\mu^{\prime} V / E^{\prime}\right)^{1 / 3} z^{2 / 3}$ was used to evaluate the integral. Note that the upper integration limit of the integral with respect to $z$ is $d$, which is the characteristic length of the problem. By equating (10) and (11), one obtains

$$
\Delta K_{I c}=\sqrt{3 \beta_{m}^{-1}} \mu^{1 / 3} E^{\prime 2 / 3} V^{1 / 3} d^{1 / 6}
$$

Note that formulas (9) and (12) give almost identical results, especially given the fact that the ratio between the corresponding numeric multipliers is $\beta_{m}^{3 / 2} \sqrt{\pi / 96} \approx 1.01$. 

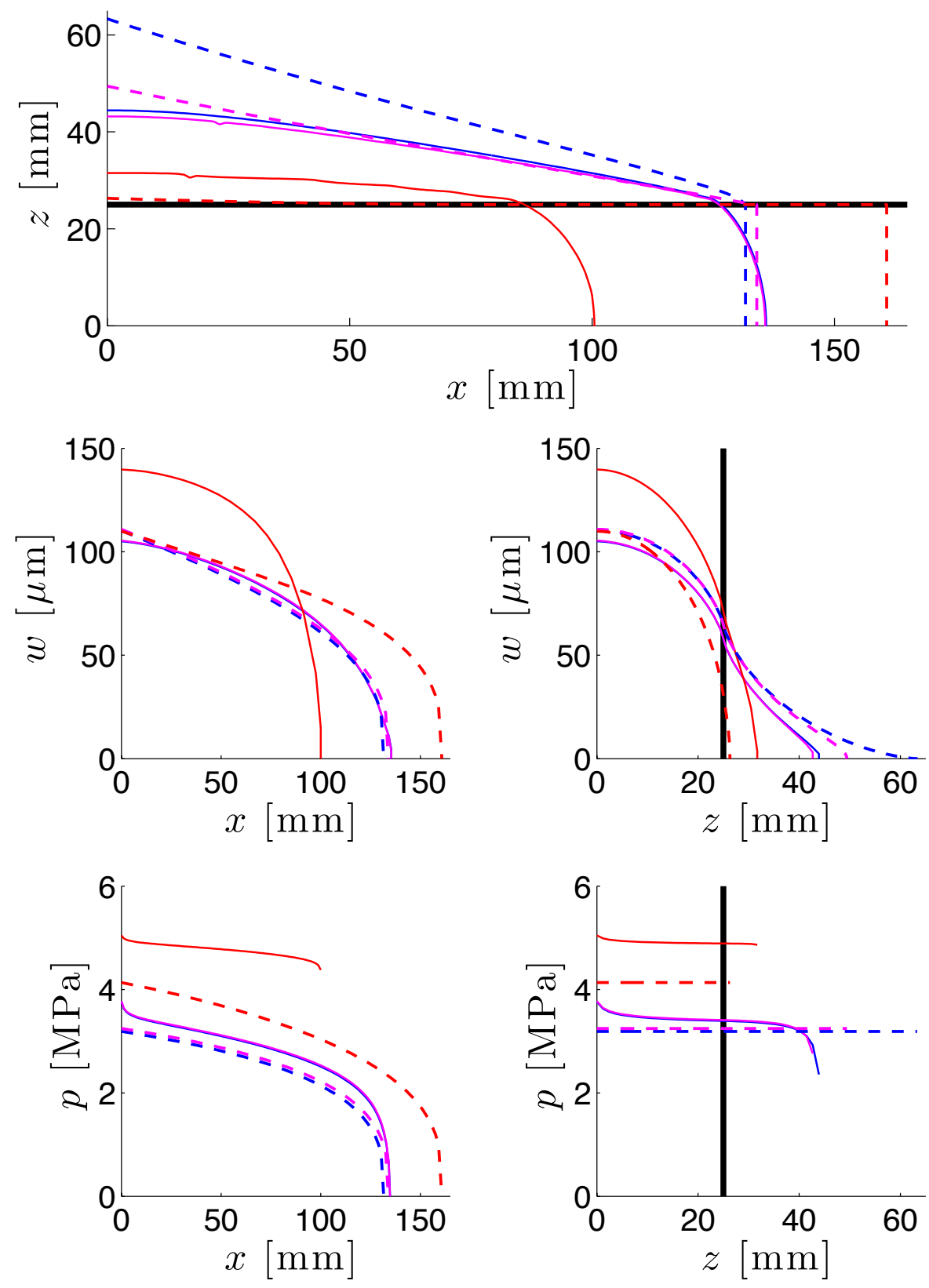

Figure 2: Comparison between the predictions of ILSA (solid lines) and the classic P3D model (dashed lines) for $K_{I c}=0$ (blue lines), $K_{I c}=0.16 \mathrm{MPa} \cdot \mathrm{m}^{1 / 2}$ (magenta lines), and $K_{I c}=0.94 \mathrm{MPa} \cdot \mathrm{m}^{1 / 2}$ (red lines). The top picture shows the various footprints, the middle pictures show the variation of the fracture opening versus $x$ at $z=0$, and versus $z$ at $x=0$. The bottom pictures show similar variations in the fluid pressure. 


\subsection{Estimating the characteristic length $d$}

To establish a framework for estimating the characteristic length $d$, first, without loss of generality, the constant multiplier is omitted in (9) and (12), which effectively redefines $d$ as

$$
\Delta K_{I c}=\mu^{1 / 3} E^{\prime 2 / 3} V^{1 / 3} d^{1 / 6} \Rightarrow d=\frac{\Delta K_{I c}^{6}}{\mu^{\prime 2} E^{\prime 4} V^{2}}
$$

To find the value of the characteristic length $d$, note that the approach of introducing an apparent toughness is also applicable to the Khristianovich-Zheltov-Geertsma-De Klerk (KGD) fracture geometry. For this reason, this subsection aims to utilize a KGD fracture geometry to find $d$. Also note that, consistent with the plane strain assumption, each vertical cross-section of a P3D fracture can be thought as a KGD fracture, see Fig. 1. Notation that is consistent with this statement is used throughout this subsection.

A symmetric KGD fracture, that is subject to stress barriers and no leak-off, is governed by the lubrication equation

$$
\frac{\partial w}{\partial t}-\frac{\partial}{\partial z}\left(\frac{w^{3}}{\mu^{\prime}} \frac{\partial p}{\partial z}\right)=\mathcal{Q}(z)
$$

and the elasticity equation, written as

$$
p-\Delta \sigma(z)=-\frac{E^{\prime}}{4 \pi} \int_{-h / 2}^{h / 2} \frac{w d s}{(s-z)^{2}},
$$

where $\mathcal{Q}(z)$ is the source term, while $\Delta \sigma(z)$ denotes the stress resulting from the stress barriers (it is zero for $|z|<H / 2$ and $\Delta \sigma$ otherwise). The boundary and propagation conditions at the fracture tip are

$$
\left.\frac{w^{3}}{\mu^{\prime}} \frac{\partial p}{\partial z}\right|_{z= \pm h / 2}=0, \quad w \rightarrow \frac{8 K_{I c}}{\sqrt{2 \pi} E^{\prime}}\left(\mp z \pm \frac{1}{2} h\right)^{1 / 2} .
$$

For KGD problems the source $\mathcal{Q}(z)$ is typically taken as $\mathcal{Q}_{0} \delta(z)$, i.e. as a point source. However, this is not the case for the P3D fracture, where the fluid for each cross-section is supplied more uniformly. Away from the well-bore the source for each KGD-like cross-section is driven by the change in the horizontal fluxes $q_{x}$ in the $x$ direction, i.e. $\partial q_{x} / \partial x$. Thus, since the pressure is assumed to be constant in each $(y, z)$ plane, it follows from Poiseuilles law that the source for each KGD-like cross section is proportional to $w^{3}(z)$. For this reason, the following distributed source is used

$$
\mathcal{Q}(z)=\mathcal{Q}_{0} \frac{w^{3}}{\int_{-h / 2}^{h / 2} w^{3} d z} .
$$

It will be shown later that the difference between the results for this distributed source and a point source is small, so the source distribution does not significantly affect the characteristic length $d$.

It is instructive to do a scaling analysis for this problem. The scales for pressure, width, length and time can be introduced respectively as

$$
p_{*}=\Delta \sigma, \quad w_{*}=\left(\frac{\mathcal{Q}_{0} \mu^{\prime} E^{\prime}}{\Delta \sigma^{2}}\right)^{1 / 2}, \quad h_{*}=\left(\frac{\mathcal{Q}_{0} \mu^{\prime} E^{\prime 3}}{\Delta \sigma^{4}}\right)^{1 / 2}, \quad t_{*}=\frac{\mu^{\prime} E^{\prime 2}}{\Delta \sigma^{3}} .
$$

In this case there are only two dimensionless parameters that determine the solution, namely, the normalized fracture toughness and the normalized height of the reservoir layer, which can be calculated as

$$
\tilde{K}_{I c}=\frac{K_{I c}}{\left(\mathcal{Q}_{0} \mu^{\prime} E^{\prime 3}\right)^{1 / 4}}, \quad \tilde{H}=\frac{H \Delta \sigma^{2}}{\sqrt{\mathcal{Q}_{0} \mu^{\prime} E^{\prime 3}}} .
$$

The procedure for finding $d$, based on the numerical calculations for the KGD fracture, can be formulated as follows. First, the numerical solution for the viscous regime (i.e. $K_{I c}=0$ ) is calculated. Then, for a 
given fracture volume $(H \bar{w})$ and fracture length $(h)$, equation $(5)$ is used to find the apparent toughness. In other words, the fracture width profile for the viscous regime is approximated by a fracture width profile that corresponds to the toughness regime by matching the fracture volume and length. Once the apparent toughness is calculated, equation (13) is used to find $d$. This approach considers an extreme case where the fracture propagates in the viscous regime. If a good approximation is obtained for this extreme case, then we would expect such apparent toughness corrections to be even more accurate for less extreme cases where some fracture toughness is present. Since the viscous solution is used, only one dimensionless parameter in (19) affects the characteristic length $d$.

We first consider a situation in which the fracture has not yet reached the stress barrier. In this situation, the parameter $\tilde{H}$ does not enter the solution, which implies that in this case $d$ depends only on the current fracture geometry, e.g. the length of the fracture $h$. Numerical calculations show that $d$ is simply proportional to $h$, i.e.

$$
d=\mathcal{C} h,
$$

where $\mathcal{C}$ is a constant. Note that the above equation could also be deduced from the fact that $h \propto t^{2 / 3}$ for a KGD fracture propagating in the viscous regime (no stress barriers) together with (5) and (13). The value of $\mathcal{C}$ obtained from numerical experiments is $\mathcal{C}=0.175$ for a distributed source $(17)$, and $\mathcal{C}=0.21$ for a point source. While this difference seems to be significant, keep in mind that the power $1 / 6$ is applied to $d$ in (13). In this case, the discrepancy becomes less than $3 \%$.

The situation when the fracture has broken through the stress barrier is more complicated, since the parameter $\tilde{H}$ affects the solution and consequently $d$. It is useful to introduce a normalized apparent toughness, which we denote by $\Delta \tilde{K}_{I c}$, where the normalization is performed according to (19), and a normalized characteristic length $\tilde{d}=d / h$. Equation (5) gives the relation between the normalized apparent toughness, $\tilde{H}$ and time, which can be written as $\Delta \tilde{K}_{I c}(\tilde{H}, t)$. At the same time, equation $(13)$ gives $\tilde{d}(\tilde{H}, t)$. Combining these expressions and inverting the solution for the fracture length $h(t)$, one may conclude that

$$
d=h \tilde{d}\left(\frac{H^{2} \Delta \sigma^{2}}{h \Delta K_{I c}^{2}}, \frac{h}{H}\right)
$$

where the first parameter signifies the ratio between $\tilde{H}^{2}$ and $\tilde{h} \Delta \tilde{K}_{I c}^{2}$, while the second parameter is the ratio between the normalized height $\tilde{h}$ and $\tilde{H}$, where the latter defines $\tilde{h}=\tilde{H} h / H$. The primary advantage of (21) lies in the fact that combining (20) with (13) yields an expression for $\Delta K_{I c}$, which does not directly depend on $\mathcal{Q}_{0}$. This makes the transition from KGD to P3D fractures easier. Given (21), there are two alternatives. Firstly, calculate $\tilde{d}$ numerically for a broad range of parameters, and then use interpolation to construct the corresponding function. Secondly, find a sufficiently accurate approximation for $\tilde{d}$. We proceed here with the second option, as it is simpler to implement and requires less computational resources, which makes the overall program faster. Let's consider the limiting case when $\Delta \sigma=0$, i.e. in which there are no stress barriers. For this situation the answer is given by (20), so that

$$
\tilde{d}\left(0, \frac{h}{H}\right)=\mathcal{C}
$$

In situations in which $H^{2} \Delta \sigma^{2} /\left(h \Delta K_{I c}^{2}\right) \gg 1$, the apparent toughness does not noticeably influence the fracture width (3), see (5) for the comparison of volumes. This can be interpreted as the "stress barrier" propagation regime, for which the resistance introduced by the stress barriers is larger than that of either the viscous and toughness resistances. The fracture geometry for this regime depends solely on the magnitude of the stress barrier and the volume of the fracture. An accurate evaluation of $\tilde{d}$ in this regime is not necessary, since the apparent toughness has little influence on the solution. For this reason, the constant approximation introduced according to (22) may already be sufficiently accurate for the complete range of expected values of the parameter $H^{2} \Delta \sigma^{2} /\left(h \Delta K_{I c}^{2}\right)$ (since it works for both limiting cases). To introduce a more accurate approximation, we try to find a characteristic distance from the fracture tip to the region where the influence of toughness is suppressed by the stress barriers. To do this, it is necessary to equate the fracture width terms in (3), one due to the toughness and the other due to $\Delta \sigma$. Since $d$ should be proportional to this 
characteristic distance, one may use the asymptotic expansion in (3), also assuming that $h / H \gg 1$, to find

$$
\tilde{d} \propto \frac{h^{1 / 2} \Delta K_{I c}}{H \Delta \sigma} \ll 1 .
$$

One of the simplest approximations that can be constructed from the above result and (22) is

$$
\tilde{d}\left(\frac{H^{2} \Delta \sigma^{2}}{h \Delta K_{I c}^{2}}, \frac{h}{H}\right) \approx \frac{\mathcal{C}}{1+\mathcal{C}_{2} \frac{H \Delta \sigma}{h^{1 / 2} \Delta K_{I c}}}
$$

where $\mathcal{C}_{2}$ is a constant.

The combination of (13), (21) and (24) leads to

$$
\Delta K_{I c}=\mathcal{C}^{1 / 6} \mu^{1 / 3} E^{\prime 2 / 3} V^{1 / 3} h^{1 / 6}\left(1+\mathcal{C}_{2} \frac{H \Delta \sigma}{h^{1 / 2} \Delta K_{I c}}\right)^{-1 / 6}
$$

which can be solved to find the apparent toughness $\Delta K_{I c}$. To check the error that is caused by the approximation (24), the numerical solution of the KGD problem (without toughness) is compared to the analytical solution (3) with the same fracture volume and the apparent toughness calculated according to (25). The numerical solution for the KGD fracture is calculated for the parameters $E^{\prime}=25 \mathrm{GPa}$, $\mu^{\prime}=1.2 \mathrm{~Pa}, \mathcal{Q}_{0}=5 \times 10^{-4} \mathrm{~m}^{2} / \mathrm{s}$ and $H=50 \mathrm{~m}$. Firstly, simulations with a maximum pumping time $t=3 \times 10^{4} \mathrm{~s}$ and various magnitudes of stress barrier $\Delta \sigma=0.25-10.5 \mathrm{MPa}$ are performed. The error, $E_{h}$, is defined as the maximum discrepancy for the fracture length for all time steps. To find the optimal value for $\mathcal{C}_{2}$, Fig. 3 shows the variation of $E_{h}$ versus $\tilde{H}$ (as this is the only parameter that affects the viscous solution of the KGD fracture with stress barriers) for different values of $\mathcal{C}_{2}$. One can observe that even with $\mathcal{C}_{2}=0$, the error is below $4 \%$, while $\mathcal{C}_{2}=1 / 2$ reduces the maximum error to less than $1.5 \%$. Note that $\mathcal{C}_{2}=1 / 2$ does not exactly correspond the the minimum of the error, but is sufficiently close that it can practically be considered a minimum. To illustrate the level of approximation of the fracture opening, Fig. 3 also shows the comparison between the widths stemming form the numerical solution (solid lines) and the approximation (3) (dashed lines) at $t=\{250,500,1000,2000,4000\} \mathrm{s}$ and $\mathcal{C}_{2}=1 / 2$. To cover different regimes, three values of the stress barrier are considered, namely, $\Delta \sigma=0.5 \mathrm{MPa}, \Delta \sigma=1.5 \mathrm{MPa}$ and $\Delta \sigma=3.5 \mathrm{MPa}$. These values correspond to $\tilde{H}=0.13$ (a weak stress barrier), $\tilde{H}=1.16$ (a moderate stress barrier), and $\tilde{H}=6.33$ (a strong stress barrier). When the stress barrier is weak, the fracture width resembles that without a stress barrier, but features a small bump at the centre. The difference between the viscous numerical solution and the approximation is visible, but not significant. When the stress barrier is strong, neither the viscosity nor the apparent toughness affect the fracture opening, in which case there is a small discrepancy between two solutions that occurs only in the near-tip regions. The case with $\tilde{H}=1.16$ represents an intermediate case, for which the fracture length error reaches a maximum.

\subsection{Application to P3D}

To apply the viscous height growth correction to the P3D model, first, the inclination of the fracture footprint located within the stress barrier is neglected. This allows us to treat each vertical cross-section of a P3D fracture as a KGD fracture and to apply the correction for the viscous resistance developed above. In terms of the numerical implementation of the P3D model with a viscous correction, the same equations (2)(7) need to be solved, in addition to the value of the apparent fracture toughness for each vertical crosssection, which needs to be calculated independently. If there is some non-zero fracture toughness, then the sum of this material fracture toughness and the apparent toughness is used in the model, i.e. $K_{I c}+\Delta K_{I c}$. To find the apparent fracture toughness at each time step, (25) can be rewritten as

$$
\Delta K_{I c}=\mathcal{C}^{1 / 6} \mu^{\prime 1 / 3} E^{\prime 2 / 3}\left(\frac{1}{2} \frac{h_{j+1}\left(K_{I c}+\Delta K_{I c}\right)-h_{j}}{t_{j+1}-t_{j}}\right)^{1 / 3} h_{j+1}^{1 / 6}\left(1+\mathcal{C}_{2} \frac{H \Delta \sigma}{h_{j+1}^{1 / 2} \Delta K_{I c}}\right)^{-1 / 6}
$$



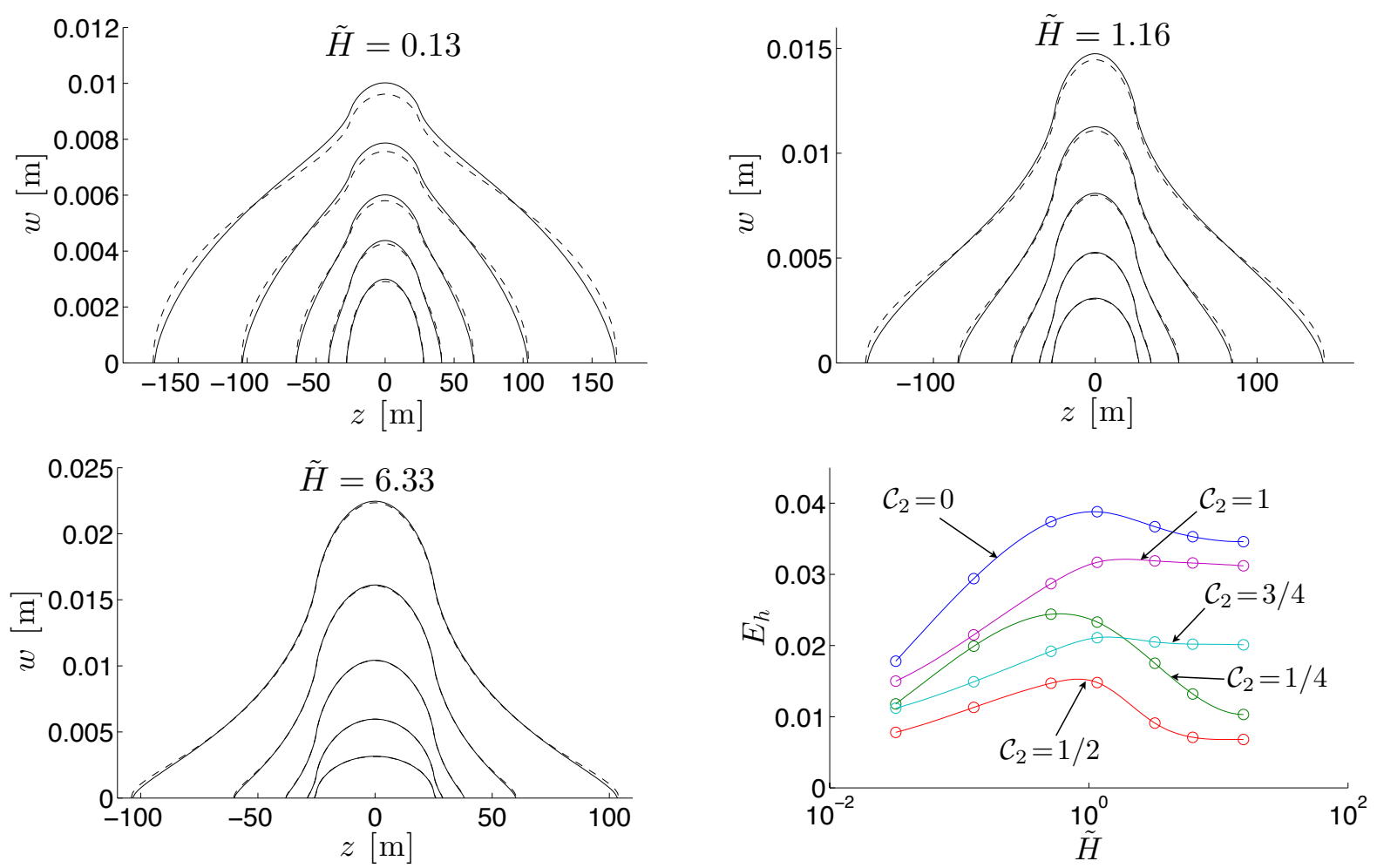

Figure 3: The top pictures and the bottom left picture show the comparison between fracture openings calculated numerically assuming no toughness (solid lines) and approximations computed using apparent toughness (dashed lines) for $t=\{250,500,1000,2000,4000\} \mathrm{s}$ and $\tilde{H}=1.16, \tilde{H}=0.13$, and $\tilde{H}=6.33$. The bottom right picture shows the fracture length error versus $\tilde{H}$ for different values of $\mathcal{C}_{2}$ (markers), the solid lines represent the spline interpolant.

where the dependence of $h$ on fracture toughness, represented by $h_{j+1}\left(K_{I c}+\Delta K_{I c}\right)$, comes from (5). Here $h_{j}$ is fracture height at some point $x$ at time $t_{i}$, while $h_{j+1}$ is an unknown fracture height at time instant $t_{j+1}$. The solution of the nonlinear equation (26) allows us to find $\Delta K_{I c}$ and consequently $h_{j+1}$ at each point in space, which are then used to solve the lubrication equation (6). The details of the numerical scheme are summarized in the Appendix. To check the accuracy of the viscous correction, Fig. 4 compares the footprints calculated using the P3D model with the viscous correction with the footprints of the reference solution given by ILSA. The parameters used for these calculations are the same as those for Fig. 2, except that the fracture toughness is $K_{I c}=0$. To show the evolution of the fracture, the footprints are plotted at different time instants, namely, $t=\{37,101,200,401,604,1048\} \mathrm{s}$. Comparison between the ILSA and P3D footprints in Fig. 4 reveals that the corrected P3D model is able to capture the height growth of the fracture even in the absence of fracture toughness. This modification thus fixes one of the major drawbacks of the P3D model, namely its inability to capture the height growth for small (or zero) values of $K_{I c}$. At the same time, for large values of the fracture toughness $\Delta K_{I c} / K_{I c} \ll 1$, the effect of the viscous correction is effectively removed.

\section{Incorporating non-local elastic interactions into P3D model}

\subsection{Non-local elasticity}

As was mentioned in Section 2.2, there are two main drawbacks of the original P3D model [26], namely, its inability to capture the height growth for a small or vanishing fracture toughness, and the significant inaccuracies for large values of $K_{I c}$, see Fig. 2. The first weakness is addressed in Section 3 through the 


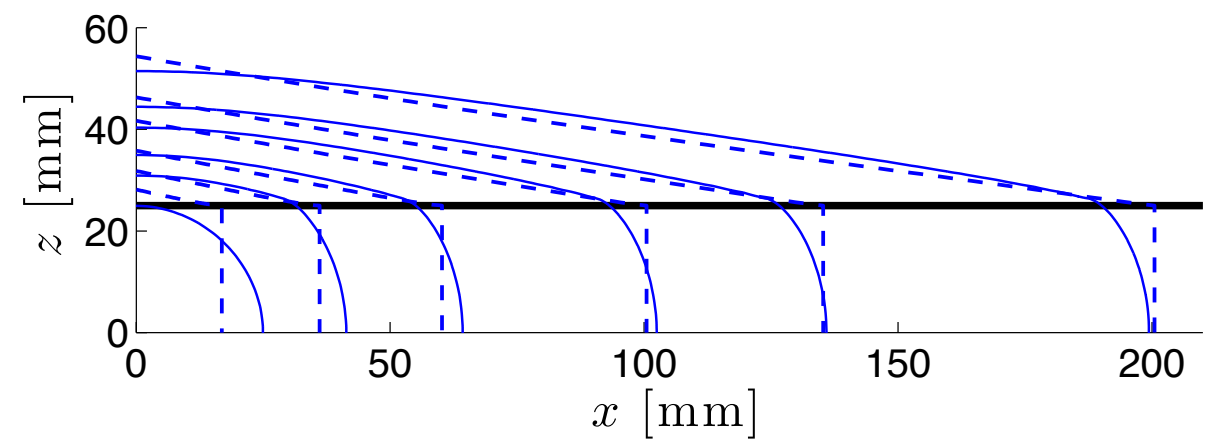

Figure 4: Comparison of fracture footprints for $K_{I c}=0$ generated by the P3D model with viscous resistance (dashed lines) and ILSA scheme (solid lines) at different time instants $t=\{37,101,200,401,604,1048\} \mathrm{s}$.

introduction of an apparent or effective fracture toughness that controls height growth, while this section aims to correct the second weak point of the P3D model by including non-local elastic interactions within the fracture. When full elastic interactions are used, formally, the pressure is no longer constant in each vertical cross-section and the fracture width cannot be calculated using the plane strain elasticity assumption (3). At the same time, Fig. 2 shows that the the fracture width variation in the vertical $z$ direction, calculated using ILSA, resembles that obtained using the P3D model - even in the viscous regime, i.e. $K_{I c}=0$. This is further supported by Fig. 3, in which the viscous KGD solutions are compared to the apparent toughness solutions. Since the difference between the viscous and apparent toughness solutions is small (as soon as the fracture height is correct), one may use the approximate analytical solution (3) to estimate the elastic interactions. In particular, the pressure relation (2) can be replaced with

$$
p(x)=-\frac{E^{\prime}}{8 \pi} \int_{-l(t)}^{l(t)} \int_{-\frac{1}{2} h\left(x^{\prime}, t\right)}^{\frac{1}{2} h\left(x^{\prime}, t\right)} \frac{w\left(x^{\prime}, z^{\prime}\right) d z^{\prime} d x^{\prime}}{\left(\left(x^{\prime}-x\right)^{2}+z^{\prime 2}\right)^{3 / 2}},
$$

where the function $w$ is given in (3), while $h$ and $l$ are the height and the half-length of the fracture respectively, see Fig. 1. Note that since the P3D model assumes that the pressure is uniform in the vertical direction $z$, only one pressure value for each vertical cross-section is required. For this reason, (27) evaluates the pressure along the $x$ axis, i.e. assumes $z=0$. For the purpose of numerical implementation, equation (27) is discretized and the integrals are evaluated numerically. This effectively gives the relation between $p(x)$ and $\bar{w}(x)$, which is analogous to the elasticity equation for the KGD fracture (15). The details of the discretization of (27) are enclosed in the Appendix. The use of (27) permits us to incorporate the asymptotic solution for the tip element, as was done in [15]. For the problem under consideration, either the viscous or the toughness asymptotic solution is used, see the Appendix for details. To illustrate the effects produced by the use of (27), Fig. 5 compares the fracture footprints generated by the P3D model with full elasticity (and the correction for the viscous resistance) to those produced by the ILSA model. The parameters used for the calculations are the same as those for Fig. 2, except that only the following two values of the fracture toughness are considered, namely, $K_{I c}=0$ and $K_{I c}=0.94 \mathrm{MPa} \cdot \mathrm{m}^{1 / 2}$. One can see that the full elasticity induces only slight changes for the viscous solution (see Fig. 4). However, the comparison between Fig. 2 and Fig. 5 shows that the use of the non-local elasticity (27) (together with the asymptotic solution for the tip element) allows us to approximate the ILSA solution extremely well for the case of large toughness. Thus, the use of the viscous correction and full elasticity allows us to overcome the two major limitations of the original P3D model, namely, its applicability for small and large values of fracture toughness.

In spite of the fact that Fig. 5 shows good agreement between the P3D model and the reference ILSA solution, another consequence of using the full elasticity is a concomitant increase in computation time. 

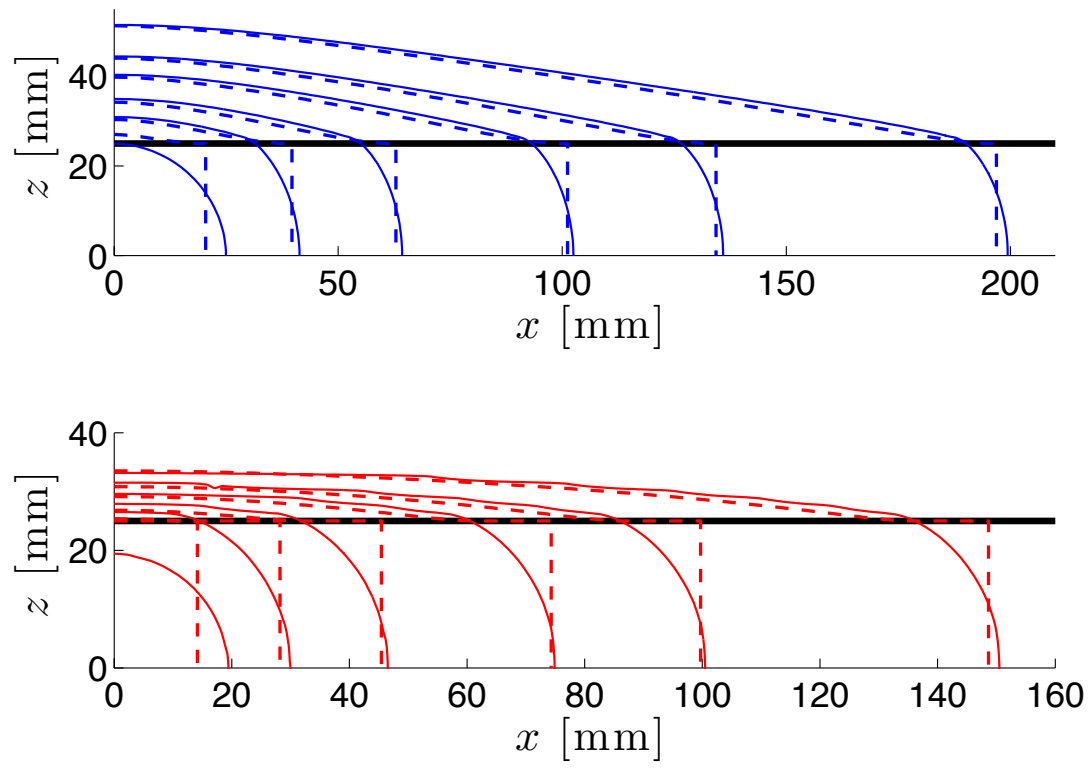

Figure 5: Comparison of the fracture footprints for $K_{I c}=0$ (top) and $K_{I c}=0.94 \mathrm{MPa} \cdot \mathrm{m}^{1 / 2}$ (bottom) generated by the P3D model with viscous resistance and full elasticity (dashed lines) and the ILSA scheme (solid lines) at different time instants $t=\{37,101,200,401,604,1048\} \mathrm{s}$.

In particular, the numerical evaluation of the double integral in (27) requires $O\left(N_{x}^{2} N_{z}\right)$ operations (recall that the pressure is evaluated at $z=0$ ), where $N_{x}$ and $N_{z}$ denote the number of elements in the $x$ and $z$ direction respectively. As a comparison, the original P3D model uses only $O\left(N_{x}\right)$ operations to evaluate pressure, a KGD model with a similar mesh requires $O\left(N_{x}^{2}\right)$ operations, while a fully planar model needs $O\left(N_{x}^{2} N_{z}^{2}\right)$ operations. This demonstrates that the introduction of non-local elasticity affects the efficiency in the computation of the pressure appreciably, and poses a significant hurdle in developing an efficient corrected P3D model. At the same time, the pressure evaluation is still much faster than that of a fully planar hydraulic fracturing model, which, together with the increased accuracy, still makes the corrected P3D model a good competitor.

\subsection{Approximate non-local elasticity}

Fig. 5 indicates that the use of the non-local elasticity significantly improves the accuracy of the P3D model, especially for large values of fracture toughness. At the same time, numerical evaluation of the double integral in (27) requires extra computational resources and slows down the overall computation time. This subsection aims to introduce an approximation to the integral (27), which would decrease time needed to calculate the pressure numerically.

First, following [25], it is noted that if one substitutes an elliptical width profile $w(x, z)=w_{0}(x) \sqrt{1-4 z^{2} / h^{2}}$ 
in $(27)$, the inner integral with respect to $z^{\prime}$ can be evaluated analytically to obtain

$$
\begin{aligned}
p(x) & =-\frac{E^{\prime}}{8 \pi} \int_{-l(t)}^{l(t)} w_{0}\left(x^{\prime}\right) \int_{-\frac{1}{2} h\left(x^{\prime}, t\right)}^{\frac{1}{2} h\left(x^{\prime}, t\right)} \frac{\sqrt{1-4 z^{\prime 2} / h^{2}} d z^{\prime}}{\left(\left(x^{\prime}-x\right)^{2}+z^{\prime 2}\right)^{3 / 2}} d x^{\prime} \\
& =\frac{E^{\prime}}{2 \pi} \int_{-l(t)}^{l(t)} \frac{w_{0}\left(x^{\prime}\right)}{h\left(x^{\prime}, t\right)\left|x^{\prime}-x\right|}\left[\mathrm{K}\left(-\frac{h\left(x^{\prime}, t\right)^{2}}{4\left(x^{\prime}-x\right)^{2}}\right)-\mathrm{E}\left(-\frac{h\left(x^{\prime}, t\right)^{2}}{4\left(x^{\prime}-x\right)^{2}}\right)\right] d x^{\prime} \\
& =\frac{E^{\prime}}{2 \pi} \int_{-l(t)}^{l(t)} \frac{w_{0}\left(x^{\prime}\right)}{h\left(x^{\prime}, t\right)-\left(x^{\prime}-x\right) \frac{\partial h\left(x^{\prime}, t\right)}{\partial x^{\prime}}} \frac{d G\left(2\left(x^{\prime}-x\right) / h\left(x^{\prime}, t\right)\right)}{d x^{\prime}} d x^{\prime},
\end{aligned}
$$

where $\mathrm{K}(\cdot)$ and $\mathrm{E}(\cdot)$ represent the complete elliptic integrals of the first and the second kind. It should be noted here that the arguments of the complete elliptic integrals are defined through the relations $\mathrm{K}(m)=$ $\int_{0}^{\pi / 2}\left(1-m \sin ^{2} \theta\right)^{-1 / 2} d \theta$ and $\mathrm{E}(m)=\int_{0}^{\pi / 2}\left(1-m \sin ^{2} \theta\right)^{1 / 2} d \theta$, and the kernel $G(\cdot)$ is given by

$$
G(s)=\frac{\sqrt{1+s^{2}}}{s} \mathrm{E}\left(\frac{1}{1+s^{2}}\right) .
$$

The asymptotic behaviour of the kernel $G(\cdot)$ is

$$
G(s) \approx \frac{\pi}{2} \operatorname{sign}(s), \quad|s| \gg 1, \quad G(s) \approx \frac{1}{s}, \quad s \ll 1 .
$$

Thus, at small distances it behaves similar to a KGD kernel, while for large distances it permits us to recover the local elasticity relation associated with the plane strain regime. Indeed, to obtain a plane strain limit, one may set $h\left(x^{\prime}\right)=$ const., $w_{0}\left(x^{\prime}\right)=$ const. and take a limit $l(t) \rightarrow \infty$ to obtain $p=E^{\prime} w_{0} /(2 h)$, which is the local pressure-width relation that is consistent with the plain strain limit.

The first term in (3) has an elliptic form and can be integrated using (28). Unfortunately, the rest of the solution, which consists of the logarithmic functions, cannot be integrated analytically. However, it can be approximated by an ellipse with some suitable parameters. To this end, let's approximate (3) by

$$
\begin{aligned}
& w(x, z) \approx w_{1}(x) \sqrt{1-4 z^{2} / h^{2}}+w_{2}(x) \sqrt{1-4 z^{2} / h_{\Delta \sigma}^{2}} \\
& w_{1}(x)=\frac{2}{E^{\prime}} \sqrt{\frac{2 h}{\pi}} K_{I c}, \quad w_{2}=\frac{2 \Delta \sigma H}{\pi E^{\prime}} \ln \left(\frac{h+\sqrt{h^{2}-H^{2}}}{h-\sqrt{h^{2}-H^{2}}}\right)
\end{aligned}
$$

where the first term, due to toughness, remains unchanged, while rest of the solution is approximated by an ellipse, whose maximum opening is the same as that for the logarithmic terms in (3), while its height $h_{\Delta \sigma}$ is taken as an unknown. It is implicitly assumed that $|z|<h / 2$ for the first term in (30), and $|z|<h_{\Delta \sigma} / 2$ for the second term in (30). To find the corresponding pressure, one needs to substitute (30) into (28), which yields

$$
\begin{aligned}
p(x) & =\frac{E^{\prime}}{2 \pi} \int_{-l(t)}^{l(t)} \frac{w_{1}\left(x^{\prime}\right)}{h\left(x^{\prime}, t\right)-\left(x^{\prime}-x\right) \frac{\partial h\left(x^{\prime}, t\right)}{\partial x^{\prime}}} \frac{d G\left(2\left(x^{\prime}-x\right) / h\left(x^{\prime}, t\right)\right)}{d x^{\prime}} d x^{\prime}, \\
& +\frac{E^{\prime}}{2 \pi} \int_{-l(t)}^{l(t)} \frac{w_{2}\left(x^{\prime}\right)}{h_{\Delta \sigma}\left(x^{\prime}, t\right)-\left(x^{\prime}-x\right) \frac{\partial h_{\Delta \sigma}\left(x^{\prime}, t\right)}{\partial x^{\prime}}} \frac{d G\left(2\left(x^{\prime}-x\right) / h_{\Delta \sigma}\left(x^{\prime}, t\right)\right)}{d x^{\prime}} d x^{\prime}
\end{aligned}
$$

To find the unknown $h_{\Delta \sigma}$, one may consider a plane strain limit of $(31)\left(h=\right.$ const., $h_{\Delta \sigma}=$ const., 
$w_{1}=$ const., $w_{2}=$ const., and $\left.l \rightarrow \infty\right)$, which gives

$$
p_{p s}=\frac{E^{\prime} w_{1}}{2 h}+\frac{E^{\prime} w_{2}}{2 h_{\Delta \sigma}}=\sqrt{\frac{2}{\pi h}} K_{I c}+\frac{\Delta \sigma H}{\pi h_{\Delta \sigma}} \ln \left(\frac{h+\sqrt{h^{2}-H^{2}}}{h-\sqrt{h^{2}-H^{2}}}\right)
$$

where $p_{p s}$ denotes the plane strain limit of $p(x)$ calculated using (31). Since, for the case of plane strain, the pressure is known and is given by (2), one may find $h_{\Delta \sigma}$ by equating this plane strain pressure and (32) to obtain

$$
h_{\Delta \sigma}=\frac{H}{\pi} \ln \left(\frac{h+\sqrt{h^{2}-H^{2}}}{h-\sqrt{h^{2}-H^{2}}}\right)\left(1-\frac{2}{\pi} \arcsin \left(\frac{H}{h}\right)\right)^{-1} .
$$

For completeness, it is noted that only one ellipse is used in situations when $h=H$, in which case $w_{1}(x)=$ $4 \bar{w}(x) / \pi$ and $w_{2}(x)=0$. The implementation of the approximation for the non-local elastic interactions requires us to replace the procedure for calculating the pressure using (27) with the approximation given by (31). More details about numerical schemes for the P3D model with non-local elasticity and with this non-local elasticity approximation are given in the appendix.

To illustrate the accuracy of the approximation, Fig. 6 compares fracture opening versus $z$ at the borehole (i.e. $x=0$ ) and the pressure variation versus $x$ calculated using the full elasticity integral (27) (dashed blue and red lines), the approximation given by (30) and (31) (solid black lines), and the reference ILSA solution (solid blue and red lines). The parameters of the problem are chosen to be the same as for the Fig. 2. Two values of fracture toughness $K_{I c}=0$ (blue lines for ILSA and full elasticity P3D) and $K_{I c}=0.94 \mathrm{MPa} \cdot \mathrm{m}^{1 / 2}$ (red lines for ILSA and full elasticity P3D) are considered, and all results are presented for time instant $t=604 \mathrm{~s}$. As can be seen from the figure, the pressure, calculated using the approximation (31), is very close to that calculated using (27). Moreover, the difference between the two is much smaller than the difference between one of them and the reference ILSA solution. This shows that the use of an approximation in the evaluation of elasticity integral does not introduce a noticeable error in the pressures. In terms of the fracture width, there is some visual difference between the two-ellipse approximation given by (30) and the P3D solution that utilizes full elasticity and whose width is calculated using (3). However, equation (3) can also be used for the purpose of calculating the fracture width of an approximate elasticity solution instead of (30). In this case, the two P3D solutions become nearly indistinguishable, similar to the pressure variations.

With regard to the numerical implementation, the pressure calculation using equation (31) requires only $O\left(N_{x}^{2}\right)$ operations, where $N_{x}$ is the number of elements in the $x$ direction. This is much smaller than the number of operations required for the evaluation of (27), which is $O\left(N_{x}^{2} N_{z}\right)$, where $N_{z}$ is the number of elements in the vertical direction. Also, this calculation shows that the computation times for the P3D model are comparable to that for a KGD fracture with the same number of elements. Note that although the calculation of the pressure is one of the slowest parts of the algorithm, there are many other operations that affect the overall computational efficiency.

\section{Curved fracture tip}

Fig. 5 shows that the use of non-local elastic interactions in a P3D model, described in Section 4.1, leads to a more accurate approximation. Moreover, Section 4.2 describes how to avoid the numerical evaluation of the elasticity integral (27), which makes the calculations faster. The P3D model with these improvements may already be sufficient for an accurate and fast hydraulic fracturing simulator. However, it it possible to improve the model even further. The modified P3D model has been improved to such an extent that the biggest discrepancies between the P3D and the reference ILSA solutions, shown in Fig. 5, are due to the flat tip assumed by the P3D model. To overcome this problem, this section aims to introduce a curved fracture tip to allow the P3D geometry to resemble the ILSA geometry more closely. Since full elasticity is used in the P3D model, and a toughness propagation criterion can be used in both the vertical and horizontal directions, it should be possible to match the solution to a radial fracture propagating in the toughness dominated regime. This naturally introduces a curved fracture tip for early times when the fracture is radial, and then a similar approach can be used later when the fracture breaks through the stress barrier. 

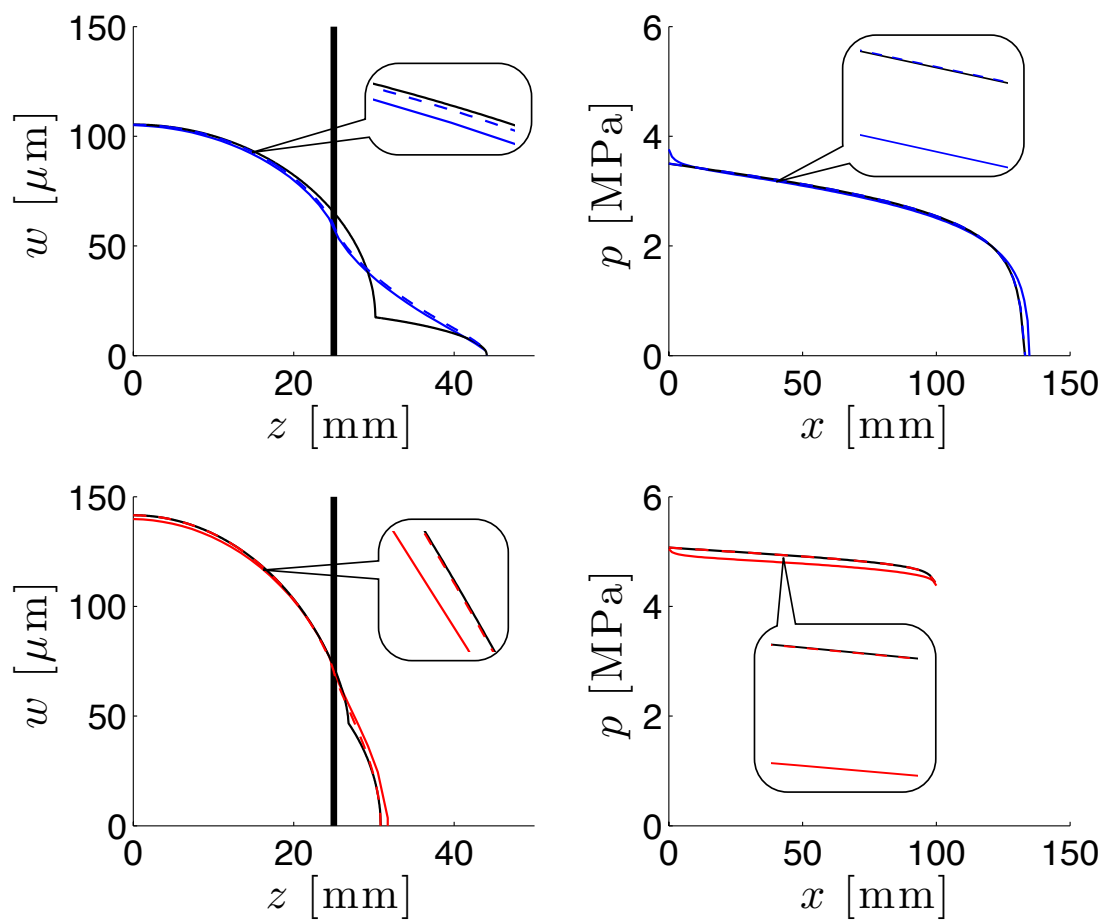

Figure 6: Comparison between the reference ILSA solution (blue and red solid lines), the P3D solution with non-local elasticity (blue and red dashed lines) and the P3D solution with approximate non-local elasticity (black lines) in terms of fracture width variation versus $z$ at $x=0$ (left column) and pressure variation versus $x$ (right column). The parameters for the computation are the same as for Fig. 2. Two values of fracture toughness $K_{I c}=0$ (top row) and $K_{I c}=0.94 \mathrm{MPa} \cdot \mathrm{m}^{1 / 2}$ (bottom row) are considered, and all results are presented for time instant $t=604 \mathrm{~s}$.

To introduce a curved fracture tip in a P3D model, we try to match the solution for a radial fracture propagating in the toughness regime. Using the same coordinate system as shown in Fig. 1, the fracture opening for the radial solution is

$$
w_{\mathrm{rad}}=\frac{4 K_{I c}}{\sqrt{\pi l} E^{\prime}} \sqrt{l^{2}-x^{2}-z^{2}},
$$

where $l$ is the half-length of the fracture and its radius at the same time. The corresponding fracture height is

$$
h_{\mathrm{rad}}=2 \sqrt{l^{2}-x^{2}} .
$$

Equation (34) can be used to calculate the effective width as

$$
\bar{w}_{\mathrm{rad}}=\frac{1}{H} \int_{-h_{\mathrm{rad}} / 2}^{h_{\mathrm{rad}} / 2} w_{\mathrm{rad}} d z=\frac{\pi K_{I c}}{2 H E^{\prime} \sqrt{\pi l}} h_{\mathrm{rad}}^{2},
$$

which can be inverted to find

$$
h_{\mathrm{rad}}=\bar{w}_{\mathrm{rad}}^{1 / 2}(2 l)^{1 / 4} \sqrt{\frac{\sqrt{2} H E^{\prime}}{\sqrt{\pi} K_{I c}}} .
$$

Equation (37) is the analog of (5), but for the radial fracture, since it relates the height of the fracture to its effective width. To find the fracture width as a function of the effective width, one may use (34), (35), 
and (36) to find

$$
w_{\mathrm{rad}}=\frac{4 H}{\pi h_{\mathrm{rad}}} \bar{w}_{\mathrm{rad}} \sqrt{1-\left(\frac{2 z}{h_{\mathrm{rad}}}\right)^{2}},
$$

which is the analog of $(3)$.

To be able to capture radial fracture propagation with the P3D model, equation (37) needs to be included into the P3D model. First, if the fracture toughness is not zero, equation (5) can only be inverted for $\bar{w}>\sqrt{\pi H / 2} K_{I c} / E^{\prime}$. For smaller $\bar{w}$, an elliptic fracture opening with the height $H$ is used in the original P3D model. This can be clearly seen in Fig. 5, where there is a region of constant fracture height near the tip for the case of non-zero toughness. To capture a radial fracture and a curved fracture tip, equations (37) and (38) should be used in this near-tip region. In situations when $2 l>H$, however, equation (37) is no longer valid since the fracture has reached the stress barrier. To ensure that the fracture height is continuous when $2 l>H$ (given the continuity of the effective width $\bar{w}$ ), equations $(5)$ and (36) should give the same result for $h=H$. This implies that the fracture height and width in the tip region should be calculated as

$$
\begin{aligned}
h & =\bar{w}^{1 / 2}(\min \{2 l, H\})^{1 / 4} \sqrt{\frac{\sqrt{2} H E^{\prime}}{\sqrt{\pi} K_{I c}}}, \\
w & =\frac{4 H}{\pi h} \bar{w} \sqrt{1-\left(\frac{2 z}{h}\right)^{2}}, \quad \bar{w}<\sqrt{\frac{\pi H}{2}} \frac{K_{I c}}{E^{\prime}},
\end{aligned}
$$

while outside of the tip region, i.e. for $\bar{w}>\sqrt{\pi H / 2} K_{I c} / E^{\prime}$, equations (5) and (3) are used. Using equations (39) in the tip region, and (3) and (5) elsewhere, ensure that the P3D fracture propagating in the toughness regime is radial at early times and features curved fracture tips later when the fracture has broken through the stress barrier.

Unlike the toughness regime of propagation, the viscous regime has no near-tip region within which the fracture height is constant and equal to $H$. This makes the introduction of a curved tip more complicated. First, a zone with constant fracture height near the tip needs to be introduced, and only then one can incorporate a curved fracture tip. To produce a zone with a constant fracture height near the tip, some effective fracture toughness, $\Delta K_{I c}$ has to be introduced. Given the effective toughness, equation (39) can be used to produce a curved tip. In situations when both the fracture toughness and the apparent toughness are relevant, (39) is replaced by

$$
\begin{aligned}
h & =\bar{w}^{1 / 2}(\min \{2 l, H\})^{1 / 4} \sqrt{\frac{\sqrt{2} H E^{\prime}}{\sqrt{\pi}\left(K_{I c}+\Delta K_{I c}\right)}}, \\
w & =\frac{4 H}{\pi h} \bar{w} \sqrt{1-\left(\frac{2 z}{h}\right)^{2}}, \quad \bar{w}<\sqrt{\frac{\pi H}{2}} \frac{K_{I c}+\Delta K_{I c}}{E^{\prime}}
\end{aligned}
$$

which is able to capture both viscous, toughness, and intermediate regimes (approximately). Even though (40) comes from the solution for a radial fracture propagating in the toughness regime, given the level of approximation of the P3D model, it can still be used for the fractures propagating in the viscous regime. The remaining question is how to find the value of the apparent toughness that would give a reasonable approximation in the viscous regime. One possibility is to introduce an apparent toughness based on the lateral propagation. In particular, a formula that is similar to (25) with $\Delta \sigma=0$, can be used, i.e.

$$
\Delta K_{I c}=\mathcal{C}_{3}^{1 / 6} \mu^{1 / 3} E^{2 / 3}\left(\frac{d l}{d t}\right)^{1 / 3} d^{1 / 6}
$$

where $\mathcal{C}_{3}$ is a constant. Equation (41) gives the value of the apparent toughness for the lateral (i.e. along $x$ axis) propagation. This apparent toughness is then used together with (40) to produce a curved fracture tip. The original formula (25) is obtained for a KGD fracture, however, it utilizes near-tip asymptotic solutions that also apply for other geometries with smooth boundaries (see [15] for a justification of this). For this 
reason, (41) can be used to calculate the effective toughness in the lateral direction. The remaining task is to determine the characteristic length $d$ and the constant $\mathcal{C}_{3}$. At early times, when the fracture should be radial, it is clear that $d=l$, i.e. equal to fracture radius. It is found through a series of numerical experiments that $\mathcal{C}_{3}=1.2$ makes the aspect ratio of the P3D-modeled fracture equal to 1 , even though the fracture is not exactly radial. Once the fracture has reached the stress barrier, ILSA simulations show that the size and shape of the region where $h<H$ does not change as the fracture propagates. This implies that the characteristic length is constant and is related to the horizontal size of the region with $h<H$, which is a small fraction of $H$. Numerical experiments show that $d=a H\left(a \approx 1.1 \times 10^{-2}\right)$ allows us to match the ILSA solutions accurately. It is clear that the characteristic length $d$ changes dramatically as the fracture breaks through the stress barrier, but it should be noted that the corresponding apparent toughness changes by less than a factor of two. To preclude a jump in the apparent toughness, a continuous transition is implemented. In particular, the characteristic distance is calculated as

$$
d=\mathcal{H}\left(\frac{1}{2} H-l\right) l+\mathcal{H}\left(l-\frac{1}{2} b H\right) a H+\mathcal{H}\left(l-\frac{1}{2} H\right) \mathcal{H}\left(\frac{1}{2} b H-l\right) \frac{\left(\frac{1}{2} b H-l\right) l+\left(l-\frac{1}{2} H\right) a H}{\frac{1}{2} b H-\frac{1}{2} H},
$$

where $\mathcal{H}$ is a Heaviside step function and $b=1.5$. Equations (40), (41) and (42) together with the values of $\mathcal{C}_{3}, a$ and $b$ allow the P3D model to capture a radial solution at early times (it is actually not exactly radial for the viscous and intermediate regimes) and introduce a curved fracture tip for fractures that have broken through the stress barrier.

The method described here has a rigorous justification for the toughness regime, while it is more empirical for the viscous and intermediate regimes, for which a number of fitting parameters are used. In addition, the introduction of a curved fracture tip is a correction, which improves the representation of the fracture geometry, and increases the overall accuracy of the P3D model.

To check the accuracy of the P3D model that is augmented with the curved tip, Fig. 7 compares the results of the corresponding P3D model (dashed lines) to the reference ILSA solutions (solid lines). The parameters for the computation are the same as for Fig. 6. Blue lines correspond to the viscous solution, i.e. with $K_{I c}=0$, while the red lines represent the results with $K_{I c}=0.94 \mathrm{MPa} \cdot \mathrm{m}^{1 / 2}$. The top two pictures compare fracture footprints at times $t=\{37,101,200,401,604,1048\} \mathrm{s}$, in which the footprints that correspond to $t=604 \mathrm{~s}$ are represented by thicker lines. The bottom four pictures are similar to the corresponding bottom four pictures of Fig. 6, as they show the comparison in terms of fracture width, w, and pressure, $p$, versus $x$ at $z=0$, and fracture width, $w$, and pressure, $p$, versus $z$ at $x=0$ at $t=604 \mathrm{~s}$. Fig. 7 shows that the P3D model with a curved fracture tip is more accurate than the equivalent P3D model with a flat tip, see Fig. 5. The fracture footprint is represented accurately from early times, when the fracture is radial, to the end of the simulation, when the fracture shape is elongated due to the presence of stress barriers. This is true for both viscous (blue lines) and toughness (red lines) regimes of propagation. The comparison of the fracture widths and pressures at $t=604 \mathrm{~s}$ further confirms the accuracy of the new P3D model. One can also observe that the constant pressure approximation in each vertical cross-section (i.e. along $z$ direction) is a reasonable assumption even for the viscous regime, since noticeable pressure deviations are localized near the source and near the fracture tip. It should be noted, that the numerical scheme for the P3D model switches between the toughness and viscous asymptotic solutions (for the tip element), so it is able to accurately represent the viscous or toughness regimes, but not the intermediate case. However, numerical simulations show that the error due to such a switch in the asymptotic solution at the tip does not exceed approximately $5 \%$ in terms of fracture length. In addition, this error can be removed by using an appropriate asymptotic solution for the M-K edge, see [29].

It is important to keep in mind the assumptions behind the modified P3D model. Clearly, since full elastic interactions are used, the fracture does not always have to be mature (i.e. elongated) for the P3D model to apply, which is confirmed in Fig. 7. At the same time, a plane-strain solution for the fracture width is used for every vertical cross-section. So, considerable discrepancies are expected in situations when the actual fracture width is not approximated accurately by a plane-strain solution. This can happen when there are both pronounced height growth through the stress barriers and the aspect ratio of the footprint is small. When both the break-through and the aspect ratio are small, the width profile is close to an ellipse, 

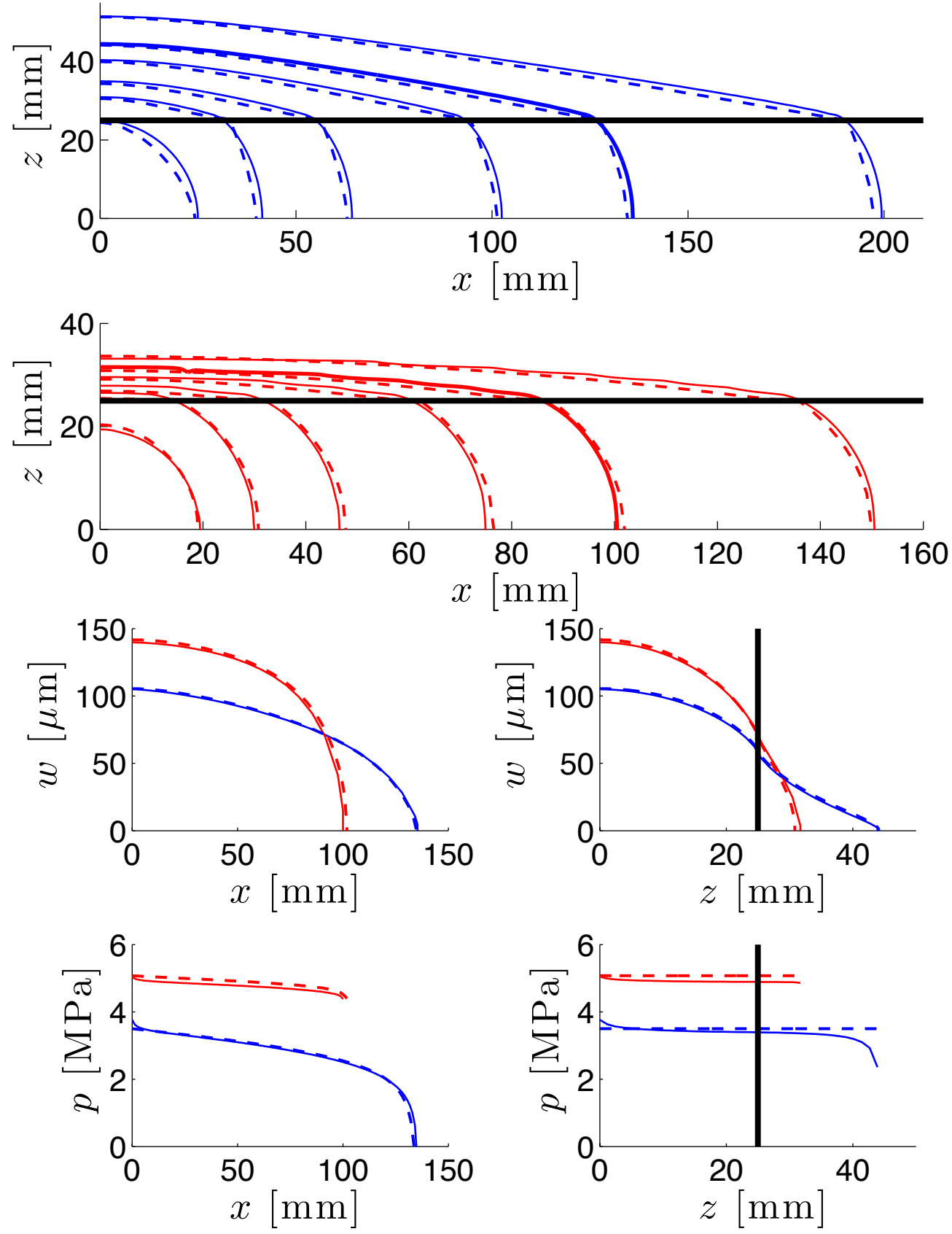

Figure 7: Comparison between the predictions of the P3D model including the approximate full elasticity and curved fracture tip (dashed lines) with the reference ILSA solutions (solid lines) for the same problem parameters as for the Fig. 6. The top two pictures compare fracture footprints at different time instants $t=\{37,101,200,401,604,1048\} \mathrm{s}$ for $K_{I c}=0$ (blue lines) and $K_{I c}=0.94 \mathrm{MPa} \cdot \mathrm{m}^{1 / 2}$ (red lines). The thick solid lines correspond to $t=604 \mathrm{~s}$. The bottom four pictures compare fracture widths and pressures versus $x$ at $z=0$ and fracture widths and pressures versus $z$ at $x=0$ at $t=604 \mathrm{~s}$. Similarly, blue lines correspond to $K_{I c}=0$, while solutions with $K_{I c}=0.94 \mathrm{MPa} \cdot \mathrm{m}^{1 / 2}$ are indicated by red lines. 

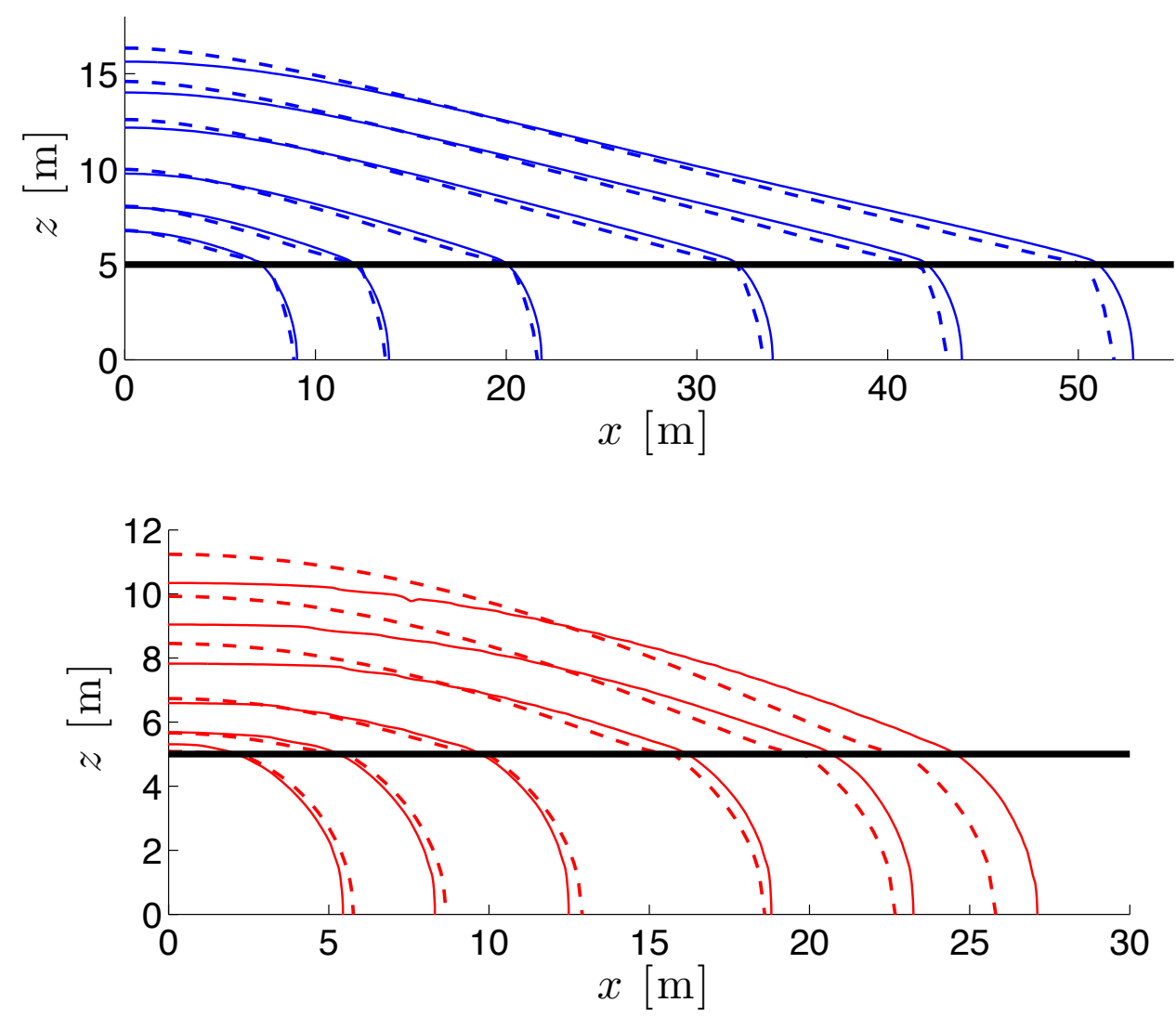

Figure 8: Comparison between the fracture footprints generated by the P3D model using a curved tip (dashed lines) and ILSA scheme (solid lines) for the set of reference parameters, Young's modulus $E=1$ GPa, $K_{I c}=0$ (top picture) and $K_{I c}=1.5 \mathrm{MPa} \cdot \mathrm{m}^{1 / 2}$ (bottom picture). Footprints are shown at time instants $t=\{51,100,204,403,599,801\} \mathrm{s}$ for the top picture and $t=\{50,100,200,399,601,801\}$ s for the bottom picture.

and the P3D model is accurate, see footprints at early times in Fig. 7. When the the fracture height growth is substantial, but the aspect ratio is high (i.e. the fracture is elongated), the conditions in each cross-section are close to plane-strain, in which case the P3D model again performs well.

To examine the accuracy of the new P3D model at the limits of its applicability, two extreme cases are considered next. The set of reference parameters for the extreme cases is $H=10 \mathrm{~m}$ for the height of the reservoir layer, $\mu=0.1 \mathrm{~Pa} \cdot \mathrm{s}$ for the fluid viscosity, $\nu=0.2$ for the Poisson's ratio, $Q_{0}=0.01 \mathrm{~m}^{3} / \mathrm{s}$ for the inlet flux and $\Delta \sigma=0.25 \mathrm{MPa}$ for the magnitude of the stress barriers. Two values of Young's modulus are considered, namely $E=1 \mathrm{GPa}$ and $E=2 \mathrm{GPa}$. In addition, two values of fracture toughness are considered, $K_{I c}=0$ and $K_{I c}=1.5 \mathrm{MPa} \cdot \mathrm{m}^{1 / 2}$. Fig. 8 shows the comparison between the fracture footprint predictions of the P3D model (dashed lines) and the ILSA solutions (solid lines) at different time instants for the case $E=1 \mathrm{GPa}$. The top picture corresponds to the case with no toughness (blue lines), while bottom picture compares the results for $K_{I c}=1.5 \mathrm{MPa} \cdot \mathrm{m}^{1 / 2}$ (red lines). As can be seen from the figure, the P3D model is accurate at early times for both the viscous and toughness regimes, while it becomes less accurate for the more mature fractures.

Moreover, the discrepancy is larger for the toughness solution, for which the aspect ratio is smaller. Since the toughness solution becomes significantly less accurate, it is clear that it is not the viscous correction 
that affects the discrepancy for the viscous solution, but the elasticity. The cause of the inaccurate elastic interactions is the use of a plane strain solution for each vertical cross-section. The errors for the fracture lengths for $t=801 \mathrm{~s}$ are $2 \%$ and $5 \%$ respectively for the viscous and toughness solutions. The errors for the fracture heights at the origin for $t=801 \mathrm{~s}$ are $5.4 \%$ and $8.5 \%$ respectively for the viscous and toughness solutions. To highlight the degree of improvement of the P3D model, this error needs to be compared with the accuracy of the original P3D model for $t=801 \mathrm{~s}$, which predicts a fracture half-length of $48 \mathrm{~m}$ and halfheight of $43 \mathrm{~m}$ for the viscous case, and a fracture half-length exceeding $80 \mathrm{~m}$ and almost no height growth for the case $K_{I c}=1.5 \mathrm{MPa} \cdot \mathrm{m}^{1 / 2}$. The even more extreme case with $E=2 \mathrm{GPa}$ is considered next. Fig. 9 shows a similar comparison between the predictions of the new P3D model (dashed lines) and the reference ILSA solutions (solid lines) for the viscous case (blue lines) and $K_{I c}=1.5 \mathrm{MPa} \cdot \mathrm{m}^{1 / 2}$ (red lines). The disagreement between the predictions of the P3D model and ILSA solutions is more pronounced compared to that in Fig. 8. This is related to the smaller aspect ratio of the footprints. In this case the plane-strain solution for the fracture width in each vertical cross-section deviates significantly from the corresponding ILSA width profile. The errors of the fracture lengths for the last considered time instant are $4 \%$ for the viscous solution and $7 \%$ for the toughness solution. The errors of the fracture heights at the origin for $t=800 \mathrm{~s}$ are $8 \%$ and $13 \%$ for the viscous and toughness solutions respectively. The magnitude of the error should be judged based on the fact that Fig. 9 represents an extreme case, which challenges the applicability of the P3D model. To establish a reference point, it is instructive to compare these results to the predictions of the original P3D model. For the viscous case, i.e. the top picture in Fig. 9, the original P3D model predicts a fracture half-length of $31.5 \mathrm{~m}$ and half-height of $293 \mathrm{~m}$ for $t=800 \mathrm{~s}$. These numbers show that the predictions of the original P3D model are very inaccurate compared to the ILSA solution, and cannot be used for any practical applications. The situation with the toughness regime, i.e. $K_{I c}=1.5 \mathrm{MPa} \cdot \mathrm{m}^{1 / 2}$, is even worse, since the original P3D model becomes unstable as soon as the height of the fracture reaches a critical value. As follows from [26], the value $h_{u}$ beyond which the classic P3D model exhibits runaway height growth, is given by

$$
h_{u}=\frac{8+\sqrt{\mathcal{K}^{4}+64}}{\mathcal{K}^{2}} H, \quad \mathcal{K}=\sqrt{\frac{2 \pi}{H}} \frac{K_{I c}}{\Delta \sigma},
$$

which is approximately equal to $h_{u} \approx 14 \mathrm{~m}$ for the considered problem parameters. As can be seen from Fig. 9, the fracture height (the ILSA solution) exceeds the critical value at early times (note that the critical half-height is $7 \mathrm{~m}$ ). Thus, due to runaway height growth, the classic P3D model fails to give a solution, while the corrected P3D model continues to give a solution albeit somewhat less accurate.

\section{Summary}

This paper aims to improve the original P3D model for hydraulic fracturing by making the predictions more accurate, but not compromising the computational efficiency. In the case of no leak-off, the primary weaknesses of the classic P3D model are its inability to capture the viscous resistance in the vertical direction and its inability to account for non-local elastic interactions. The latter, in particular, precludes capturing the toughness propagation regime in the lateral direction. To include the viscous resistance in the vertical direction, a toughness solution with an apparent toughness is used. To obtain an expression for the appropriate apparent toughness, a similar approach is applied to a one-dimensional KGD hydraulic fracturing model, where a fracture propagating in the viscous regime is approximated by a solution that corresponds to the toughness regime and features an apparent toughness. A comparison with the results obtained using the fully planar HF simulator ILSA show that the introduction of an apparent toughness allows us to capture fracture height growth accurately using the P3D model even when there is no toughness. To account for the toughness propagation criterion in the lateral direction, non-local elasticity is introduced. First, the fracture width in every cross-section is approximated by a plane strain solution, and then the appropriate elasticity integral is evaluated numerically. This approach also allowed us to include the asymptotic solution in the tip element, which makes it possible to capture fracture propagation in either the viscous or the toughness regimes. Comparison with the ILSA results demonstrate that the use of non-local elasticity significantly increases the accuracy of the P3D model, especially when the lateral fracture propagation is in the toughness 

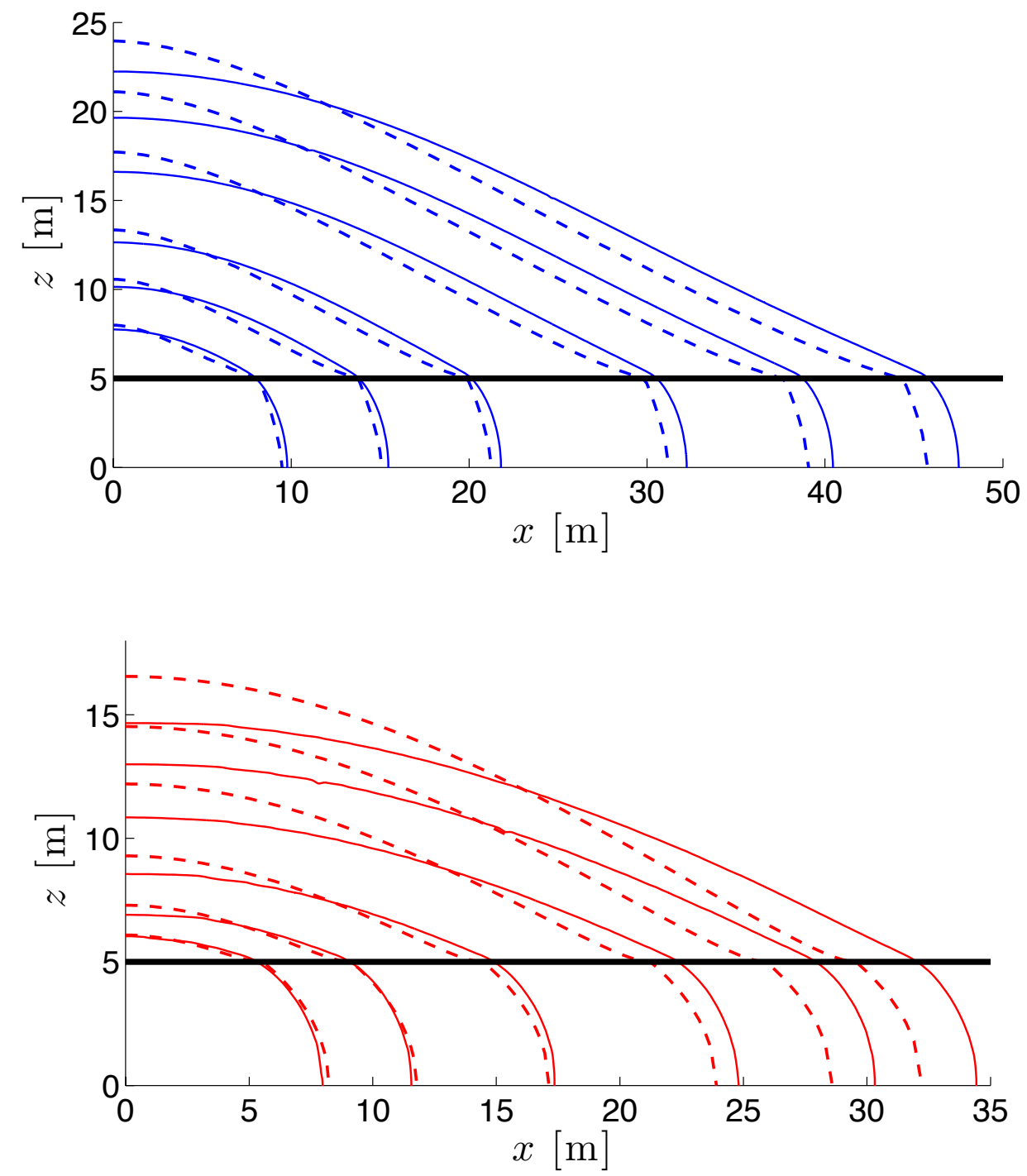

Figure 9: Comparison between fracture footprints generated by the P3D model using a curved tip (dashed lines) and ILSA scheme (solid lines) for the set of reference parameters, Young's modulus $E=2$ GPa, $K_{I c}=0$ (top picture) and $K_{I c}=$ $1.5 \mathrm{MPa} \cdot \mathrm{m}^{1 / 2}$ (bottom picture). Footprints are shown for time instants $t=\{51,112,203,401,600,800\} \mathrm{s}$ for the top picture and $t=\{52,99,201,401,600,801\}$ s for the bottom picture. 
regime.To make the computation of non-local elastic interactions faster, the fracture opening in the vertical direction is approximated by two ellipses, for each of which the elasticity integral is calculated analytically over the vertical axis. This effectively reduces the computation of the 2D integral to two 1D integrals, which makes the resulting P3D model significantly more efficient. The last improvement of the P3D model lies in replacing the flat fracture tip by a curved tip. To do this, the P3D model is tuned to match the analytic solution for a radial fracture propagating in the toughness regime. In this case, mature fractures (i.e. those that have reached the stress barriers) feature a tip region, in which the properties of a radial fracture dictate the shape of the fracture tip.

A radial fracture propagating in the viscous regime is also accounted for approximately by introducing an apparent toughness in the lateral direction. The accuracy of the resulting enhanced P3D model is verified by comparing the results to the predictions of the fully planar HF simulator ILSA. It is shown that the P3D model is able to capture the evolution of hydraulic fractures from the initial stages, when the fracture is radial, to mature stages when the fracture has broken through the stress barrier. Moreover, this evolution can be captured when fractures propagate in either the viscous or toughness regimes. To examine the limits of the applicability, extreme cases are considered. Even for such extreme parameters, the accuracy of the enhanced P3D model is on the order of $10 \%$, while the error of the original P3D model is measured in hundreds of percent.

\section{Acknowledgements}

The authors gratefully acknowledge the support of the British Columbia Oil and Gas Commission and the NSERC discovery grants program.

\section{Appendix: Numerical scheme}

This appendix aims to describe the numerical solution for the P3D model with all its variations, i.e. starting from the original model with plane strain elasticity, and finishing with the model that captures full elastic interactions together with the curved fracture tip. For all cases, it is assumed that there is no leak-off and that a point source is used to represent the wellbore. To effectively deal with the moving boundary problem, a scaled $x$ coordinate is introduced, $\xi=x / l(t)(0 \leqslant \xi \leqslant 1)$, where $l(t)$ is a half-length of the fracture. In this case, equation (6) can be rewritten as

$$
\frac{\partial \bar{w}}{\partial t}-\frac{\xi}{l} \frac{d l}{d t} \frac{\partial \bar{w}}{\partial \xi}+\frac{1}{l} \frac{\partial \bar{q}_{x}}{\partial \xi}=\frac{Q_{0}}{H l} \delta(\xi) .
$$

where the averaged flux is given by

$$
\bar{q}_{x}=-\frac{1}{H l \mu^{\prime}} \frac{\partial p}{\partial \xi} \int_{-h / 2}^{h / 2} w^{3} d z .
$$

Equations (43) and (44) are common for all methods, while the differences come from the procedures for calculating $w, h$ and $p$ based on the values of $\bar{w}$. For the purpose of the numerical computations, both the spatial variable $\xi$ and the temporal variable $t$ are discretized. Uniform discretization in $\xi$ is used, i.e. $\xi_{i}=i / N_{\xi}, i=0 \ldots N_{\xi}, \Delta \xi=1 / N_{\xi}$, where $N_{\xi}$ is number of spatial discretization points. At the same time, nonuniform temporal discretization is used to address the fact that fractures tend to grow rapidly at earlier stages requiring a smaller time step, and then slow down significantly later in the simulation allowing for coarser time increments.

To achieve the nonuniform discretization of the temporal variable that is consistent with the features of the problem, the time step is taken so that $t_{j+1}-t_{j} \propto t_{j}^{\gamma}$, where $j=0 \ldots N_{t}$ and $\gamma$ is some power that is between 0 and 1 . Here $N_{t}$ is number of time steps and $\gamma$ is chosen based on the regime of propagation. The choice of power law-type behaviour is based on the fact that the fracture length and width are known to obey power-law time dependence in some significant regimes of propagation. By using $\bar{w}_{i}^{j}=\bar{w}\left(\xi_{i}, t_{j}\right)$ to denote 


\begin{tabular}{c|c|c|c} 
& Computation of $h$ & Computation of $w$ & Condition \\
\hline Inner region & Invert (5) & Use $(3)$ & $\bar{w}>\sqrt{\frac{\pi H}{2}} \frac{K_{I c}}{E^{\prime}}$ \\
Tip region & $h=H$ & $w=\frac{2}{E^{\prime}} \sqrt{H^{2}-4 z^{2}} p$ & $\bar{w} \leqslant \sqrt{\frac{\pi H}{2}} \frac{K_{I c}}{E^{\prime}}$
\end{tabular}

Table 1: Calculation of fracture height $h$ and fracture width $w$ for different parameters in the original P3D model.

the approximation for the effective fracture width and $\bar{q}_{x, i \pm \frac{1}{2}}^{j}=\bar{q}_{x}\left(\xi_{i} \pm \frac{\Delta \xi}{2}, t_{j}\right)$ to denote the approximation for the fluxes, equation (43) can be discretized at point $\xi_{i}$ as

$$
\frac{\bar{w}_{i}^{j+1}-\bar{w}_{i}^{j}}{t_{j+1}-t_{j}}-\frac{\xi_{i+\frac{1}{2}} V\left(t_{j+1}\right)}{l\left(t_{j+1}\right)} \frac{\bar{w}_{i+1}^{j+1}-\bar{w}_{i-1}^{j+1}}{2 \Delta \xi}+\frac{1}{l\left(t_{j+1}\right)} \frac{\bar{q}_{x, i+\frac{1}{2}}^{j+1}-\bar{q}_{x, i-\frac{1}{2}}^{j+1}}{\Delta \xi}=\frac{Q_{0}}{H l\left(t_{j+1}\right)} \frac{\delta_{i 0}}{\Delta \xi},
$$

where $V\left(t_{j+1}\right)=d l / d t$ is the velocity of propagation of the fracture, while $\delta_{i 0}$ is Kronecker delta that ensures that the source belongs exclusively to the element with index 0 located at the origin. Equation (45) features a backward time difference, in which case its solution utilizes an appropriate iterative scheme at each time step to solve the nonlinear system of algebraic equations (45). In spite of this complexity, the L-stable backward Euler scheme allows us to avoid stability issues and to use an arbitrary time step, see [15] in which a similar approach is used. Two boundary conditions are used, namely, $\bar{w}_{N_{\xi}}^{j+1}=0$, and $\left.\bar{q}_{x}\right|_{\xi=1}=0$. In addition, a symmetry condition $\bar{q}_{x,-\frac{1}{2}}^{j+1}=-\bar{q}_{x, \frac{1}{2}}^{j+1}$ is utilized. For completeness, it is noted that the fracture length is updated using $l\left(t_{j+1}\right)=l\left(t_{j}\right)+V\left(t_{j+1}\right)\left(t_{j+1}-t_{j}\right)$. The difference in implementation of various P3D models lies in computation of $V\left(t_{j+1}\right)$ and the fluxes $\bar{q}_{x, i \pm \frac{1}{2}}^{j+1}$ as a function of $\bar{w}$.

Original P3D model.. To complete the scheme for the numerical implementation of the original P3D model, the fluxes need to be prescribed. To do so, firstly the fracture height needs to be evaluated. There are two cases. First, when $\bar{w}>\sqrt{\pi H / 2} K_{I c} / E^{\prime}$, equation (5) is inverted to find $h$. In situations when $\bar{w} \leqslant$ $\sqrt{\pi H / 2} K_{I c} / E^{\prime}$, a constant height $h=H$ is used. The fracture opening, appearing in (44) is taken from (3) for the first case, while if $h=H$, the following elliptic opening is chosen $w=2\left(E^{\prime}\right)^{-1} \sqrt{H^{2}-4 z^{2}} p$. This procedure is summarized in Table 1. Using this aforementioned procedure, the integral in (44) is evaluated numerically using the trapezoidal rule for each spatial discretization point. To evaluate the pressure gradient, it is noted that in the case $h>H$, equations (2) and (5) can be used to compute

$$
\frac{\partial p}{\partial \xi}=\frac{d p}{d h} \frac{d h}{d \bar{w}} \frac{\partial \bar{w}}{\partial \xi}=Y(h) \frac{\partial \bar{w}}{\partial \xi}
$$

where

$$
Y(h)=\frac{H E^{\prime}}{\pi h^{2}} \frac{4 \Delta \sigma h^{1 / 2}-\sqrt{2 \pi} K_{I c} \sqrt{\frac{h^{2}}{H^{2}}-1}}{3 \sqrt{\frac{\pi}{2}} K_{I c} \sqrt{\frac{h^{2}}{H^{2}}-1}+2 \Delta \sigma h^{1 / 2}} .
$$

This formula automatically reduces to $\partial p / \partial \xi=2 E^{\prime} /(\pi H) \partial \bar{w} / \partial \xi$ for the case $h=H$. The fluxes are computed using (46) as

$$
\bar{q}_{x, i+\frac{1}{2}}^{j+1}=-\frac{1}{H l\left(t_{j+1}\right) \mu^{\prime}} Y\left(h\left(\xi_{i+\frac{1}{2}}\right)\right) \frac{\bar{w}_{i+1}^{j+1}-\bar{w}_{i}^{j+1}}{\Delta \xi} \sum_{k=0}^{N_{z}} w^{3}\left(l\left(t_{j+1}\right) \xi_{i+\frac{1}{2}}, z_{k}\right) \Delta z
$$

where the sum appears from the numerical integration. Here the $z$ coordinate is discretized as $z_{k}=-\frac{h}{2}+\Delta z k$, $k=0 \ldots N_{z}$, where $\Delta z=h / N_{z}$, so that different element sizes are used for each cross-section (since $h$ varies 
with $\xi$ ). The quantities that need to be evaluated at the mid points (i.e. at $\xi_{i+\frac{1}{2}}$ ) are treated using linear interpolation. To find the fracture velocity, equation (43) is integrated over the tip element, which, together with the zero flux boundary condition, allows us to find

$$
V\left(t_{j+1}\right)=\frac{\bar{q}_{x, N_{\xi}-\frac{1}{2}}^{j+1}}{\bar{w}_{i+\frac{1}{2}}^{j+1}\left(1-\frac{1}{2} \Delta \xi\right)} .
$$

Using (47) and (48), the resulting system of nonlinear algebraic equations (45) is solved iteratively for each time step for $\bar{w}_{i}^{j+1}, i=0 \ldots N_{\xi}$. Once the effective width, $\bar{w}$, is calculated, formulas (5) and (3) allow us to find fracture footprint and opening.

Original P3D model with the correction for viscous height growth.. The implementation of the correction for the viscous resistance does not require many modifications. First, equation (26) can be rewritten as

$$
\Delta K_{I c, i}^{j+1}=\mathcal{C}^{1 / 6} \mu^{\prime 1 / 3} E^{\prime 2 / 3}\left(\frac{1}{2} \frac{h_{i}^{j+1}-\tilde{h}_{i}^{j}}{t_{j+1}-t_{j}}\right)^{1 / 3}\left(h_{i}^{j+1}\right)^{1 / 6}\left(1+\mathcal{C}_{2} \frac{H \Delta \sigma}{\left(h_{i}^{j+1}\right)^{1 / 2} \Delta K_{I c, i}^{j+1}}\right)^{-1 / 6}
$$

Here $\Delta K_{I c, i}^{j+1}$ denotes the correction to the fracture toughness at point $\xi_{i}$, while $h_{i}^{j+1}$ are calculated from (5), where the fracture toughness is replaced by $K_{I c}+\Delta K_{I c, i}^{j+1}$. Since the moving mesh is used, $\tilde{h}_{i}^{j}$ is not just $h_{i}^{j}$, because the calculated velocity is not exactly vertical in this case. Since the physical position $x$ has to be fixed during the calculation of the vertical velocity, one needs to take $\tilde{h}_{i}^{j}=\mathcal{I}\left[h_{i}^{j}\right]\left(l\left(t_{j+1}\right) \xi_{i}\right)$, where the latter means the interpolation of $h_{i}^{j}\left(i=0 \ldots N_{\xi}\right)$ at the point $l\left(t_{j+1}\right) \xi_{i}$. The values of the constants in (49), which are used for the computations, are $\mathcal{C}=0.175$ and $\mathcal{C}_{2}=0.5$. The solution of the nonlinear equation (49) together with $h_{i}^{j+1}$ coming from (5), obtained using Newton's method, produces both $h_{i}^{j+1}$ and $\Delta K_{I c, i}^{j+1}$. Finally, the fracture width, $w$, is calculated by replacing $K_{I c}$ with $K_{I c}+\Delta K_{I c, i}^{j+1}$ in (3). With these modifications in the calculation of $h$ and $w$, the solution is obtained by solving equation (45) together with (47) and (48).

P3D with non-local elasticity.. The implementation of non-local elasticity requires more significant modifications. First, since the pressure is evaluated at $z=0$, there should be an element located at this point, which requires $N_{z}$ to be even (so that the total number of elements $N_{z}+1$ is odd). With this meshing, equation (27) can be discretized as

$$
p_{i}^{j}=\left(\tilde{C}_{i k m}^{j}+\tilde{C}_{i(-k) m}^{j}\right) W_{k m n}^{j} \bar{w}_{n}^{j}=C_{i n}^{j} \bar{w}_{n}^{j},
$$

where index "-k" means evaluation at negative $\xi_{k}$, which allows us to build symmetry into the influence coefficients $C_{i n}^{j}$ (to avoid capturing element with $k=0$ twice, $\tilde{C}_{i(-k) m}^{j}$ is added only for $k \geqslant 1$ ),

$$
\begin{aligned}
\tilde{C}_{i k m}^{j} & =-\frac{E^{\prime} l\left(t_{j}\right)}{8 \pi} \int_{\xi_{k}-\frac{1}{2} \Delta \xi}^{\xi_{k}+\frac{1}{2} \Delta \xi} \int_{z_{m}-\frac{1}{2} \Delta z}^{z_{m}+\frac{1}{2} \Delta z} \frac{d z^{\prime} d \xi^{\prime}}{\left(l\left(t_{j}\right)^{2}\left(\xi^{\prime}-\xi_{i}\right)^{2}+z^{\prime 2}\right)^{3 / 2}} \\
& =-\frac{E^{\prime}}{8 \pi}\left[\frac{\sqrt{l\left(t_{j}\right)^{2}\left(\xi_{i}-\xi^{\prime}\right)^{2}+z^{\prime 2}}}{l\left(t_{j}\right)\left(\xi_{i}-\xi^{\prime}\right) z^{\prime}}\right]_{\xi^{\prime}=\xi_{k}-\frac{1}{2} \Delta \xi, z^{\prime}=z_{m}-\frac{1}{2} \Delta z}^{\xi^{\prime}=\xi_{k}+\frac{1}{2} \Delta \xi, z^{\prime}=z_{m}+\frac{1}{2} \Delta z},
\end{aligned}
$$

and

$$
W_{k m n}^{j}=\frac{w^{j}\left(l\left(t_{j}\right) \xi_{k}, z_{m}\right)}{\bar{w}_{k}^{j}} \delta_{k n}
$$


Here $w^{j}\left(l \xi_{k}, z_{m}\right)$ in (52) denotes the evaluation of the fracture opening according to (3) (or ellipse) at $x=l\left(t_{j}\right) \xi_{k}$ and $z=z_{m}$ and $\delta_{k n}$ is the Kronecker delta. Relations (51) and (52) permit the computation of the elasticity matrix $C_{i n}^{j}$ featured in (50). Note, however, that this elasticity matrix is a nonlinear function of $\bar{w}_{n}^{j}$, where this nonlinear dependence comes implicitly through the variation of $h$ and explicitly from (52). The fluxes are calculated using the discretization of (44) as

$$
\bar{q}_{x, i+\frac{1}{2}}^{j+1}=-\frac{1}{H l\left(t_{j+1}\right) \mu^{\prime}} \frac{p_{i+1}^{j+1}-p_{i}^{j+1}}{\Delta \xi} \sum_{k=0}^{N_{z}} w^{3}\left(l\left(t_{j+1}\right) \xi_{i+\frac{1}{2}}, z_{k}\right) \Delta z
$$

where the values of the pressure are now calculated using (50).

Unfortunately, calculating the pressure using the hypersingular integral (27) leads to poor accuracy for the tip element. To deal with this, the asymptotic solution can be built into the tip element, as was done in [15]. To do so, it is assumed that the tip element follows the asymptotic solution (obtained for KGD fracture), and the fracture propagation velocity is calculated accordingly. In particular, if the fracture propagates in the viscous regime, the velocity of propagation is calculated as

$$
V\left(t_{j+1}\right)=\frac{E^{\prime}}{\mu^{\prime}} \frac{w^{3}\left(l\left(t_{j+1}\right)(1-\Delta \xi), 0\right)}{\beta_{m}^{3}\left(l\left(t_{j+1}\right) \Delta \xi\right)^{2}},
$$

where $\beta_{m}=2^{1 / 3} \cdot 3^{5 / 6}$ and $w\left(l\left(t_{j+1}\right)(1-\Delta \xi), 0\right)$ is the fracture opening at $z=0$ for the penultimate node. If fracture propagates in the toughness regime, the velocity is adjusted in a way that the toughness propagation criterion holds for the last element, i.e.

$$
w\left(l\left(t_{j+1}\right)(1-\Delta \xi), 0\right)=\sqrt{\frac{32}{\pi}} \frac{K_{I c}}{E^{\prime}} \sqrt{l\left(t_{j+1}\right) \Delta \xi} .
$$

In situations when the fracture propagates in the intermediate regime, one of the asymptotic solutions is used. The criterion for switching between the two solutions is the following: at the beginning, the velocity of propagation is calculated for both viscous and toughness regimes, and then the minimum velocity is chosen. Physically, there are two resistance mechanisms - viscous and toughness. Taking the minimum velocity implies that only the dominant resistance is considered. In situations when both mechanisms contribute, the velocity, and consequently the fracture length, are overestimated. Since fractures typically evolve from the viscous regime to the toughness regime (assuming no leak-off), the error introduced during the transition zone is mitigated later when the toughness regime dominates. Moreover, comparison with ILSA simulations shows that even during the intermediate regime, the length was overestimated only by approximately $5 \%$.

To compensate for the introduction of the extra condition comprising the formula for the velocity of fracture propagation, and to ensure a zero flux boundary condition at the tip, the pressure at the tip element needs to be treated as an unknown, as was also done in [15]. Knowing the velocity of propagation, equation (48) can be used to find the tip pressure, $p_{N_{\xi}}^{j+1}$, as

$$
p_{N_{\xi}}^{j+1}=p_{N_{\xi}-1}^{j+1}-\frac{H l\left(t_{j+1}\right) \mu^{\prime} \Delta \xi V\left(t_{j+1}\right) \bar{w}_{N_{\xi}-\frac{1}{2}}^{j+1}\left(1-\frac{1}{2} \Delta \xi\right)}{\sum_{k=0}^{N_{z}} w^{3}\left(l\left(t_{j+1}\right) \xi_{N_{\xi}-\frac{1}{2}}, z_{k}\right) \Delta z} .
$$

Finally, the algorithm with full elasticity consists of solving (45), where the fluxes are computed using (53), the pressure (for all nodes, except the tip element) is calculated using (50)-(52), the fracture propagation velocity is taken such that either (54) or (55) is satisfied depending on the regime of propagation, while the pressure at the tip element is taken from (56). It should also be noted that the algorithm for calculating the apparent toughness, based on (49), is included in its original form, as it is independent of the type of elasticity equation that is used for calculations. 


\begin{tabular}{c|c|c|c|c} 
& Computation of $h$ & Computation of $w$ & Computation of $\Delta K_{I c}$ & Condition \\
\hline Inner region & Invert (5) & Use (3) & Use (49) & $\bar{w}>\sqrt{\frac{\pi H}{2}} \frac{K_{I c}+\Delta K_{I c}}{E^{\prime}}$ \\
Tip region & Use (40a) & Use (40b) & Use (41) & $\bar{w} \leqslant \sqrt{\frac{\pi H}{2}} \frac{K_{I c}+\Delta K_{I c}}{E^{\prime}}$
\end{tabular}

Table 2: Calculation of the fracture height $h$, the fracture width $w$, and the apparent toughness $\Delta K_{I c}$ for different parameters for the P3D model with curved tip.

P3D with approximate non-local elasticity.. The only difference between the P3D algorithm with an approximate non-local elasticity operator from the one described above, which includes full elasticity, lies in calculation of elasticity matrix $C_{i n}^{j}$ in (50). By approximating both $h$ and $\bar{w}$ as piece-wise constant functions, equation (31) can be reduced to

$$
p_{i}^{j}=\left[\left(C_{1, i k}^{j}+C_{1, i(-k)}^{j}\right) W_{1, k n}^{j}+\left(C_{2, i k}^{j}+C_{2, i(-k)}^{j}\right) W_{2, k n}^{j}\right] \bar{w}_{n}^{j}=\hat{C}_{i n}^{j} \bar{w}_{n}^{j},
$$

where $\hat{C}_{i n}^{j}$ is an approximation to $C_{i n}^{j}$, index " $-k$ " denotes the evaluation of $C_{1, i k}^{j}$ at $-\xi_{k}$ in order to capture symmetry (as before, to avoid capturing element with $k=0$ twice, terms with " $-k$ " are added only for $k \geqslant 1)$, and

$$
\begin{aligned}
& C_{1, i k}^{j}=\left.\frac{E^{\prime}}{2 \pi} G\left(\frac{2 l\left(t_{j}\right)\left(\xi^{\prime}-\xi_{i}\right)}{h\left(l\left(t_{j}\right) \xi^{\prime}, t_{j}\right)}\right)\right|_{\xi^{\prime}=\xi_{k}-\frac{1}{2} \Delta \xi} ^{\xi^{\prime}=\xi_{k}+\frac{1}{2} \Delta \xi}, \quad W_{1, k n}^{j}=\frac{w_{1}\left(l\left(t_{j}\right) \xi_{k}, t_{j}\right)}{h\left(l\left(t_{j}\right) \xi_{k}, t_{j}\right) \bar{w}_{k}} \delta_{k n}, \\
& C_{2, i k}^{j}=\left.\frac{E^{\prime}}{2 \pi} G\left(\frac{2 l\left(t_{j}\right)\left(\xi^{\prime}-\xi_{i}\right)}{h_{\Delta \sigma}\left(l\left(t_{j}\right) \xi^{\prime}, t_{j}\right)}\right)\right|_{\xi^{\prime}=\xi_{k}-\frac{1}{2} \Delta \xi} ^{\xi^{\prime}=\xi_{k}+\frac{1}{2} \Delta \xi}, \quad W_{2, k n}^{j}=\frac{w_{2}\left(l\left(t_{j}\right) \xi_{k}, t_{j}\right)}{h_{\Delta \sigma}\left(l\left(t_{j}\right) \xi_{k}, t_{j}\right) \bar{w}_{k}} \delta_{k n} .
\end{aligned}
$$

The kernel $G$ is defined in (29), $w_{1}$ and $w_{2}$ as a functions of $h$ are given in (30), while $h_{\Delta \sigma}$ is defined in (33). In situations when $h=H, w_{1}\left(l\left(t_{j}\right) \xi_{k}, t_{j}\right)=4 \bar{w}_{k}^{j} / \pi$ and $w_{2}=0$. Evaluation of $w_{1}\left(l\left(t_{j}\right) \xi_{k}, t_{j}\right), w_{2}\left(l\left(t_{j}\right) \xi_{k}, t_{j}\right)$, and $h_{\Delta \sigma}\left(l\left(t_{j}\right) \xi_{k}, t_{j}\right)$ all require the evaluation of $h\left(l\left(t_{j}\right) \xi_{k}, t_{j}\right)$, which in turn depends on $\bar{w}\left(l\left(t_{j}\right) \xi_{k}, t_{j}\right)=\bar{w}_{k}^{j}$. In this case, similar to the full elasticity matrix, the approximate elasticity matrix, $\hat{C}_{i n}^{j}$, is a nonlinear function of $\bar{w}_{k}^{j}$. Note that since a piece-wise constant approximation for $h$ is used (because $\bar{w}$ is also piece-wise constant), spatial derivatives of $h$ and $h_{\Delta \sigma}$ in (31) do not influence the result.

The P3D model with the approximate full elasticity requires the solution of the same equations as the model for the full elasticity, except that the subroutine for evaluating the fluid pressure (50)-(52) is replaced by one corresponding to (57)-(58).

Curved fracture tip.. To introduce a curved fracture tip, the algorithm needs to be updated in several places. The computation of the fracture height, $h$, and the width in the tip region is now performed using (40). Table 2 summarizes the procedure and replaces the corresponding procedure for the original P3D model that is summarized in Table 1. In addition to the different formulas for $h$ and $w$, the apparent toughness $\Delta K_{I c}$ is evaluated differently. In particular, equation (41) is used to find the fracture toughness in the tip region. The evaluation of the fluid pressure using (57) and (58) has two modifications. First, since $h<H$ in the tip region and only one ellipse is used to approximate the fracture width, $w_{1}\left(l\left(t_{j}\right) \xi_{k}, t_{j}\right)=4 H /\left(\pi h\left(l\left(t_{j}\right) \xi_{k}, t_{j}\right)\right) \bar{w}_{k}^{j}$ and $w_{2}=0$. Unfortunately, since $\partial h / \partial x$ in (31) is singular at the tip element (if a curved fracture tip is used), the piece-wise constant approximation for $h$ precludes an accurate evaluation of the elasticity integral. To deal with this problem, we seek a correction that makes the elasticity integral accurate. One way to estimate the accuracy of the elasticity equation is to consider a radial fracture propagating in the toughness regime. Since, in this case, every vertical cross-section is an ellipse the radial fracture can be accurately represented by the P3D model with a curved fracture tip. If one uses (35) and (36), and evaluates the fluid pressure using (57) and (58), then the resultant pressure should be constant (since the fracture propagates in the 

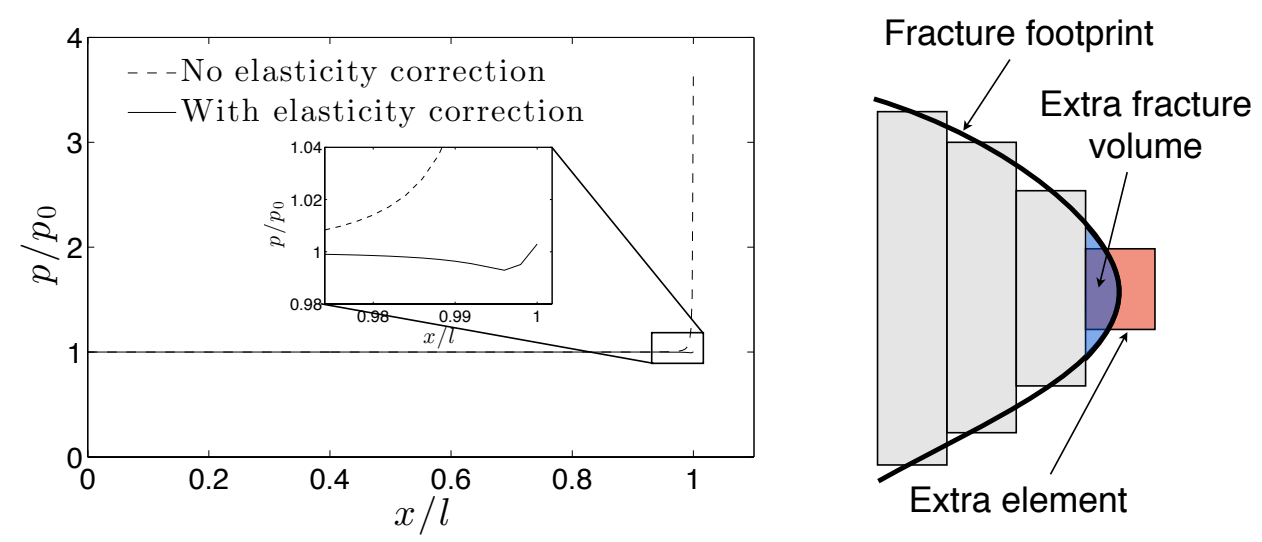

Figure 10: Variation of the normalized pressure versus the normalized distance for a radial fracture propagating in the toughness regime, calculated numerically using (57) with and without the correction (left picture). The right picture schematically shows the location of the extra element that is used for the correction.

toughness regime). All deviations from the constant in this case are due to the inaccuracy caused by the evaluation of the elasticity equation. The results of implementing this procedure show that the calculated pressure deviates from a constant near the tip and so a correction is required, see the dashed line in Fig. 10. Note that there is a fracture volume at the tip that is not accounted for in the pressure calculations. In other words, since the fracture opening is zero at the tip, the opening of the corresponding element is zero and so this element does not contribute to the pressure. To introduce a correction, one may account for the extra volume by introducing a non-zero tip element for pressure calculations, see Fig. 10. By assuming that the fracture opening follows the toughness asymptotic solution for the tip element, it can be shown that the volume of the extra element is $1 / 8$ of that of penultimate element. Then, to determine both $h$ and $w_{1}$ for the extra element, its height is taken as $h_{N_{\xi}}=h_{N_{\xi}-1} / r$, where $r=1.825$ is a constant. The value of $r$ is found by minimizing the error between the numerically calculated pressure and the constant (which corresponds to the exact solution). Fig. 10 shows that the fluid pressure inside the radial fracture, that is calculated using the extra tip element, is accurate everywhere to within $1 \%$ error.

To summarize, the numerical scheme for the P3D model with a curved tip with the approximate non-local elasticity and correction for viscous height growth consists of solving (45), where the fluxes are computed using (53), the pressure (for all nodes, except the tip element) is calculated using (57) and (58), the fracture propagation velocity is taken such that either (54) or (55) is satisfied depending on the regime of propagation, while the pressure at the tip element is taken from (56). Procedures for calculating the fracture height, width, as well as the apparent toughness are summarized in Table 2.

Initial condition. Even though the initial condition does not affect the solution in the long term, it is important to specify an appropriate initial condition, since, otherwise, the numerical algorithm may not converge at the first time step. For both viscous and toughness regimes of propagation it is assumed that at a given small time instant $t_{1}$ the hydraulic fracture is radial and the fracture opening corresponds to the solution for "dry" crack, i.e. given by

$$
w_{\mathrm{rad}}=\frac{4\left(K_{I c}+\Delta K_{I c}\right)}{\sqrt{\pi l} E^{\prime}} \sqrt{l^{2}-x^{2}-z^{2}}
$$

where the apparent toughness $\Delta K_{I c}$ is calculated based on (41). By calculating the volume of the fracture from (59) and equating it to the pumped volume (no leak-off), we obtain

$$
Q_{0} t=\frac{8}{3} \frac{\sqrt{\pi}\left(K_{I c}+\mathcal{C}_{3}^{1 / 6} \mu^{1 / 3} E^{2 / 3} l^{1 / 6} \frac{d l^{1 / 3}}{d t}\right) l^{5 / 2}}{E^{\prime}} .
$$


The latter differential equation (60) is solved numerically to find $l\left(t_{1}\right), d l\left(t_{1}\right) / d t$, and $\Delta K_{I c}\left(t_{1}\right)$. Note that the differential equation reduces to an algebraic equation in the toughness regime, in which case

$$
l\left(t_{1}\right)=\left(\frac{3 E^{\prime} Q_{0} t_{1}}{8 \sqrt{\pi} K_{I c}}\right)^{2 / 5}, \quad \frac{d l}{d t}\left(t_{1}\right)=\frac{2}{5}\left(\frac{3 E^{\prime} Q_{0}}{8 \sqrt{\pi} K_{I c}}\right)^{2 / 5} t_{1}^{-3 / 5}, \quad \Delta K_{I c}\left(t_{1}\right)=0 .
$$

Having $l\left(t_{1}\right), d l\left(t_{1}\right) / d t$, and $\Delta K_{I c}\left(t_{1}\right)$, the effective width is calculated from (59) as

$$
\bar{w}\left(t_{1}\right)=\frac{2 \sqrt{\pi}\left(K_{I c}+\Delta K_{I c}\left(t_{1}\right)\right) l\left(t_{1}\right)^{3 / 2}}{H E^{\prime}}\left(1-\xi^{2}\right),
$$

while, since the fracture is radial, its height is given by

$$
h\left(t_{1}\right)=2 l\left(t_{1}\right) \sqrt{1-\xi^{2}} .
$$

This procedure for calculating the initial condition is exact if the fracture propagates in the toughness regime, while it gives an approximate initial condition if the fracture propagates in the viscous regime. One may also consider using the solution for a radial fracture propagating in the viscous regime [31]. However, since the P3D model can represent such a fracture only approximately, it is sufficient to use an approximation for the initial condition as well.

\section{References}

[1] U. Frank, N. Barkley, Remediation of low permeability subsurface formations by fracturing enhancements of soil vapor extraction, J. Hazard. Mater. 40 (2005) 191-201.

[2] A. Abou-Sayed, D. Andrews, I. Buhidma, Evaluation of oily waste injection below the permafrost in prudhoe bay field, in: Proceedings of the California Regional Meetings, Bakersfield, CA, Society of Petroleum Engineers, Richardson, TX, 1989, pp. 129-142.

[3] R. Jeffrey, K. Mills, Hydraulic fracturing applied to inducing longwall coal mine goaf falls, in: Pacific Rocks 2000, Balkema, Rotterdam, 2000, pp. 423-430.

[4] M. Economides, K. Nolte (Eds.), Reservoir Stimulation, 3rd Edition, John Wiley \& Sons, Chichester, UK, 2000.

[5] S. Khristianovic, Y. Zheltov, Formation of vertical fractures by means of highly viscous fluids, in: Proc. 4th World Petroleum Congress, Vol. 2, 1955, pp. $579-586$.

[6] T. Perkins, L. Kern, Widths of hydraulic fractures, in: J. Pet. Tech. Trans. AIME, 1961, pp. 937-949.

[7] R. Nordgren, Propagation of vertical hydraulic fractures, in: J. Pet. Technol., 1972, pp. 306-314.

[8] I. P. ID, H. Carroll, Three-dimensional hydraulic fracture propagation in the presence of stress variation, in: Proceedings of the SPE/DOE/GRI unconventional gas recovery symposium, SPE/DOE 10849, 1982, pp. 870-878.

[9] I. P. ID, H. Carroll, Numerical solution for height and elongated hydraulic fractures, in: Proceedings of the SPE/DOE low permeability reservoir symposium, Denver. SPE 11627, 1983, pp. 249-256.

[10] A. Settari, M. Cleary, Development and testing of a pseudo-three-dimensional model of hydraulic fracture geometry (p3dh), in: Proceedings of the 6th SPE symposium on reservoir simulation of the Society of Petroleum Engineers, SPE 10505, 1982, pp. 185-214.

[11] J. McLennan, J. Picardy, Pseudo-three-dimensional fracture growth modeling, in: 26th US Symposium on Rock Mechanics, 1985, pp. 323-331.

[12] Y. Xiujuan, W. Tongtao, Y. Xiangzhen, W. Xin, A pseudo-3D model with 2D flow of hydraulic fracture propagation, in: Thin Interbedded Sandstone Reservoir. International Society for Rock Mechanics., 2010, pp. 429-433.

[13] S. H. A. T. Lee, J. Lee, Three-dimensional modeling of hydraulic fractures in layered media: Part I-finite element formulations, J. Energy Resour. Technol. 112 (1990) 1-9.

[14] L. Vandamme, J. Curran, A three-dimensional hydraulic fracturing simulator, Int. J. Numer. Meth. Eng. 28 (1989) 909927.

[15] A. Peirce, E. Detournay, An implicit level set method for modeling hydraulically driven fractures, Comput. Methods Appl. Mech. Engrg. 197 (2008) 2858-2885.

[16] J. Adachi, E. Siebrits, A. Peirce, J. Desroches, Computer simulation of hydraulic fractures, Int. J. Rock Mech. Min. Sci. 44 (2007) 739-757.

[17] L. Germanovich, L. R. D. Astakhov, J. Shlyopobersky, M. Mayerhofer, Hydraulic fracture with multiple segments II: Modeling, Int. J. Rock Mech. Min. Sci. 34 (1997) 472.

[18] A. Peirce, A. Bunger, Interference fracturing: Non-uniform distributions of perforation clusters that promote simultaneous growth of multiple hydraulic fractures, SPE 172500.

[19] J. A. L. Napier, E. Detournay, Propagation of non-planar pressurized cracks from a borehole, in: Research and Applications in Structural Engineering, Mechanics and Computation, 2013, pp. 597-602. 
[20] M. Yang, Hydraulic fracture production optimization with a pseudo-3D model in multilayered lithology, in: Society of Petroleum Engineers, SPE 149833, 2012.

[21] W. Kordziel, W. Rowe, V. Dolan, S. Ritger, A case study of integrating well-logs and a pseudo 3D multi-layer frac model to optimize exploitation of tight lenticular gas sands, in: Society of Petroleum Engineers, SPE 36866, 1996.

[22] R. Wu, O. Kresse, X. Weng, C.-E. Cohen, H. Gu, Modeling of interaction of hydraulic fractures in complex fracture networks, in: Society of Petroleum Engineers, SPE 152052, 2012.

[23] X. Weng, Incorporation of 2D fluid flow into a pseudo-3D hydraulic fracturing simulator, in: Society of Petroleum Engineers, 1992, pp. 331-337.

[24] E. Sarvaramini, D. Garagash, Pressurization of a PKN Fracture in Permeable Rock during Injection of a Low Viscosity Fluid, Tech Publishers, 2013, Ch. 30, pp. 629-640.

[25] J. Adachi, A. Peirce, Asymptotic analysis of an elasticity equation for a finger-like hydraulic fracture, J. Elasticity 90 (2008) 43-69.

[26] J. I. Adachi, E. Detournay, A. P. Peirce, An analysis of classical pseudo-3D model for hydraulic fracture with equilibrium height growth across stress barriers, Int. J. of Rock Mech. and Min. Sci. 47 (2010) 625-639.

[27] E. Carter, Optimum fluid characteristics for fracture extension, in: Howard GC, Fast CR, editors. Drilling and production practices, 1957, pp. 261-270.

[28] E. Detournay, Propagation regimes of fluid-driven fractures in impermeable rocks, Int. J. Geomech. 4 (2004) $35-45$.

[29] A. Peirce, Modeling multi-scale processes in hydraulic fracture propagation using the implicit level set algorithm, (accepted) Comp. Meth. in Appl. Mech. and Eng.

[30] R. Jeffrey, A. Bunger, A detailed comparison of experimental and numerical data on hydraulic fracture height growth through stress contrasts, in: Society of Petroleum Engineers, SPE 106030, 2007.

[31] A. Savitski, E. Detournay, Similarity solution of a penny-shaped fluid-driven fracture in a zero-toughness linear elastic solid, CR. Acad. Sci. II B 329 (2001) 255-262. 